\title{
New Weighted Norm Inequalities for Certain Classes of Multilinear Operators and their Iterated Commutators
}

\author{
Guixia $\operatorname{Pan}^{1} \cdot$ Lin Tang $^{2}$
}

Received: 7 June 2012 / Accepted: 22 March 2015 / Published online: 9 April 2015

(C) Springer Science+Business Media Dordrecht 2015

\begin{abstract}
Applying the new class of multiple weights functions and new sharp maximal functions, we obtain the pointwise estimates, strong type and weak end-point estmates for certain classes of multilinear operators and their iterated commutators with new BMO functions.
\end{abstract}

Keywords Multilinear operator · Multiple weight

Mathematics Subject Classification (2010) 42B25 - 42B20

\section{Introduction}

Let $T$ be a multilinear operator initially defined on the $m$ - fold product of Schwarz spaces and take values into the space of tempered distributions,

$$
T: \mathbb{S}\left(\mathbb{R}^{n}\right) \times \ldots \times \mathbb{S}\left(\mathbb{R}^{n}\right) \rightarrow \mathbb{S}^{\prime}\left(\mathbb{R}^{n}\right) .
$$

The research was supported by the NNSF (11271024) of China.

Lin Tang

tanglin@math.pku.edu.cn

Guixia Pan

pgxkd@163.com

1 School of Public Health, Anhui Medical University,

Anhui, 230032, People's Republic of China

2 LMAM, School of Mathematical Sciences, Peking University,

Beijing, 100871, People's Republic of China 
We study that $T$ is a multilinear operator and satisfies the following conditions:

(1) If there exists a function $K$, defined off the diagonal $x=y_{1}=\ldots=y_{m}$ in $\left(\mathbb{R}^{n}\right)^{m+1}$, such that

$$
T\left(f_{1}, \ldots, f_{m}\right)(x)=\int_{\left(\mathbb{R}^{n}\right)^{m}} K\left(x, y_{1}, \ldots, y_{m}\right) f_{1}\left(y_{1}\right) \ldots f_{m}\left(y_{m}\right) d y_{1} \ldots d y_{m}
$$

for all $x \notin \cap_{j=1}^{m}$ supp $f_{j}$;

(2) For any $N \geq 0$, there exists $C>0$ such that

$$
\left|K\left(y_{0}, y_{1}, \ldots, y_{m}\right)\right| \leq \frac{C}{\left(\sum_{k, l=0}^{m}\left|y_{k}-y_{l}\right|\right)^{m n}\left(1+\sum_{k, l=0}^{m}\left|y_{k}-y_{l}\right|\right)^{N}} ;
$$

(3) For some $\varepsilon>0$ and any $N \geq 0$, there exists $C>0$ such that

$$
\begin{aligned}
\mid K\left(y_{0}, \ldots, y_{j}, \ldots,\right. & \left.y_{m}\right)-K\left(y_{0}, \ldots, y_{j}^{\prime}, \ldots, y_{m}\right) \mid \\
\leq & \frac{C\left|y_{j}-y_{j}^{\prime}\right|^{\varepsilon}}{\left(\sum_{k, l=0}^{m}\left|y_{k}-y_{l}\right|\right)^{m n+\varepsilon}\left(1+\sum_{k, l=0}^{m}\left|y_{k}-y_{l}\right|\right)^{N}},
\end{aligned}
$$

provided that $0 \leq j \leq m$ and $\left|y_{j}-y_{j}^{\prime}\right| \leq \frac{1}{2} \max _{0 \leq k \leq m}\left|y_{j}-y_{k}\right|$;

(4) There exist $1 \leq q_{1}, \cdots, q_{m}<\infty$ and $\frac{1}{q}=\frac{1}{q_{1}}+\cdots+\frac{1}{q_{m}}$ such that $T$ is bounded from

$$
L^{q_{1}} \times \ldots \times L^{q_{m}} \rightarrow L^{q} .
$$

When $N=0$ in Eqs. 1.2 and 1.3, such kernels are called m-linear Calderón-Zygmund kernels. since the multilinear operator $T$ satisfying (1.1)-(1.4) has better properties than an m-linear Calderón-Zygmund operator, from [7] we know that

$$
T: L^{q_{1}} \times \cdots \times L^{q_{m}} \rightarrow L^{q}
$$

where $\frac{1}{q}=\frac{1}{q_{1}}+\cdots+\frac{1}{q_{m}}, 1<q_{j}<\infty$ for all $j=1, \cdots, m$; and

$$
T: L^{q_{1}} \times \cdots \times L^{q_{m}} \rightarrow L^{q, \infty},
$$

where $1 \leq q_{1}, \cdots, q_{m}<\infty$ and $\frac{1}{q_{1}}+\ldots+\frac{1}{q_{m}}=\frac{1}{q}$. In particular, it will be relevant the case

$$
T: L^{1} \times \cdots \times L^{1} \rightarrow L^{\frac{1}{m}, \infty} .
$$

If the operator $T$ satisfies (1.1)-(1.4), we know that multilinear pseudodifferential operator is only the special case of $T$; see $[1,2,14]$.

The commutator of a linear Calderón-Zygmund operator $T$ and a BMO function $b$,

$$
T_{b}(f)=[b, T](f)=b T(f)-T(b f),
$$

was first studied by Coifman, Rochberg, and Weiss [5] who proved that

$$
T_{b}: L^{p}\left(\mathbb{R}^{n}\right) \rightarrow L^{p}\left(\mathbb{R}^{n}\right),
$$


actually

$$
\left\|T_{b}(f)\right\|_{L^{p}} \leq C\|b\|_{B M O}\|f\|_{L^{p}} .
$$

Let $\vec{b}=\left(b_{1}, \ldots, b_{m}\right)$ be in $B M O^{m}$. The commutator of $\vec{b}$ and the $m$ - Linear Calderón-Zygmund operator $T$, denoted here by $T_{\Sigma \vec{b}}$, was introduced by Pérez and Torres in [12] and is defined via

$$
T_{\Sigma \vec{b}}\left(f_{1}, \ldots, f_{m}\right)=\sum_{j=1}^{m} T_{b_{j}}^{j}\left(f_{1}, \ldots, f_{m}\right),
$$

where each term is the commutator of $b_{j}$ and $T$ in the $j$-th entry of $T$, that is,

$$
T_{b_{j}}^{j}(\vec{f})=\left[b_{j}, T\right](\vec{f}) \equiv b_{j} T\left(f_{1}, \ldots, f_{j}, \ldots, f_{m}\right)-T\left(f_{1}, \ldots, b_{j} f_{j}, \ldots, f_{m}\right) .
$$

It was shown in [12] that $T_{\Sigma \vec{b}}$ satisfies the bounds (1.5) for all indices satisfying $\frac{1}{q}=$ $\frac{1}{q_{1}}+\cdots+\frac{1}{q_{m}}$ with $1<q_{j}<\infty$ for all $j=1, \cdots, m$ and $q>1$; The result was extended in [8] to all $q>\frac{1}{m}$. The estimates are of the form

$$
\left\|T_{\Sigma \vec{b}}(\vec{f})\right\|_{L^{q}} \leq C\left(\sum_{j=1}^{m}\left\|b_{j}\right\|_{B M O}\right) \prod_{j=1}^{m}\left\|f_{j}\right\|_{L^{q_{j}}} .
$$

Moreover, weighted- $L^{p}$ versions of the bounds (1.5) were obtained in [8] for weights in the class $A_{\vec{p}}$ (see again the definitions in the next section). The end-point estimate (1.6) does not hold for $T_{\Sigma} \vec{f}$. Instead the following estimate was also obtained in [8]

$$
\left|\left\{x \in \mathbb{R}^{n}:\left|T_{\Sigma \vec{b}}(\vec{f})(x)\right|>t^{m}\right\}\right| \leq C(\vec{b}) \prod_{j=1}^{m}\left(\int_{\mathbb{R}^{n}} \Phi\left(\frac{\left|f_{j}(x)\right|}{t}\right) d x\right)^{\frac{1}{m}},
$$

where $\Phi(t)=t\left(1+\log ^{+} t\right)$. The result is still true if the Lebesgue measured is changed by an $A \rightarrow$ weight.

For a multilinear operator $T$ and $\vec{b}=\left(b_{1}, \ldots, b_{m}\right)$ in $B M O_{\vec{\theta}}^{m}$ (see the definition in the next section), we define the iterated commutators $T_{\Pi \vec{b}}$ to be

$$
T_{\Pi \vec{b}}\left(f_{1}, \ldots, f_{m}\right)=\left[b_{1},\left[b_{2}, \ldots\left[b_{m-1},\left[b_{m}, T\right]_{m}\right]_{m-1} \ldots\right]_{2}\right]_{1}(\vec{f}) .
$$

To clarify the notation, if $T$ is associated in the usual way with a kernel $K$ satisfying (1.1), (1.2) and (1.3), then at a formal level

$T_{\Pi \vec{b}}(\vec{f})(x)=\int_{\mathbb{R}^{n m}} \prod_{j=1}^{m}\left(b_{j}(x)-b_{j}\left(y_{j}\right)\right) K\left(x, y_{1}, \ldots, y_{m}\right) f_{1}\left(y_{1}\right) \ldots f_{m}\left(y_{m}\right) d y_{1} \ldots d y_{m}$.

Recently, Lener, etc, [8] applied the new maximal functions and multiple weights to study the weighted norm inequalities of multilinear Calderón-Zygmund operators and their commutators with BMO functions. Futhermore, Përe, etc, [11] gave end-point estimates for iterated commutators of multilinear singular integrals. Very recently, Tang [13] obtained that weighted $L^{p}$ inequalities for pseudo-differential operators with smooth symbols and their commutators by using a class of new weight functions. In addition, Bongioanni, etc, [3] proved $L^{p}\left(\mathbb{R}^{n}\right)(1<p<\infty)$ boundedness for commutators of Riesz transforms associated with Schrödinger operator with $B M O_{\theta}(\rho)$ functions which include the class $B M O$ function, and they [4] established the weighted boundedness for some Schrödinger type operators with weight $A_{p}^{\rho, \theta}$ class which includes the weight class in [13]. 
Inspired by the works above, in this paper we focus on the weighted boundedness of certain classes of multilinear operators for the new class of multiple weights and their iterated commutators with the new BMO functions.

The article is organized as follows. Section 2 contains some basic definitions and facts concerning on a new class of multiple weights, new sharp maximal functions, and Orlicz spaces needed throughout the rest of this work. In Section 3, we give the pointwise and strong(weak)-type estimates for maximal operators and multilinear operators. Finally, we give the pointwise and strong(weak)-type estimates for multilinear iterated commutators in Section 4.

Remark During preparing this paper, we learned that Bui [2] obtained the weighted boundedness of the operators T satisfying (1.1)-(1.4) and commutators generated by T satisfying (1.1)-(1.4) and the new BMO function in the multiple weights setting (see Corollaries 3.2 and 4.2). It should be pointed out that, in this paper, we not only give these results by using a different way posed in [13] (see also [9, 10]), but also we give some new results such as the end-point estimates for commutators.

\section{Some Preliminaries and Notations}

We first recall some notations. $Q(x, r)$ denotes the cube centered at $x$ with the sidelength $r$ and $\lambda Q(x, r)=Q(x, \lambda r)$. Given a Lebesgue measurable set $E,|E|$ will denote the Lebesgue measure of $E$. For $m$ exponents $p_{1}, \ldots, p_{m}$, we will often write $p$ for the number given by $\frac{1}{p}=\frac{1}{p_{1}}+\ldots+\frac{1}{p_{m}}, \vec{p}$ for the vector $\vec{p}=\left(p_{1}, \ldots, p_{1}\right)$ and $\vec{\theta}=\left(\theta_{1}, \ldots, \theta_{m}\right)$. For any number $r>0, r \vec{p}$ is defined by $r \vec{p}=\left(r p_{1}, \ldots, r p_{m}\right)$. In this paper, we write $\varphi(Q)=(1+r)$, where $r$ is the sidelength of $Q$ and $\theta>0$.

\subsection{The New Class of Weights}

In this section, we recall the definition of the new class of weights introduced by [4].

For $1 \leq p<\infty$ and $\theta \geq 0$, the weight $\omega$ ( $\omega$ is a nonnegative and locally integrable function) is said to be in the class $A_{p}^{\theta}$ if there holds

$$
\left(\int_{Q} \omega\right)^{\frac{1}{p}}\left(\int_{Q} \omega^{\frac{-1}{p-1}}\right)^{\frac{1}{p^{\prime}}} \leq C|Q|(1+r)^{\theta}
$$

for all cube $Q(x, r)$. In particular case when $p=1$, is understood

$$
\frac{1}{|Q|} \int_{Q} \omega(y) d y \leq C(1+r)^{\theta} \inf _{x \in Q} \omega(x) .
$$

Then we denote $A_{p}^{\infty}=\cup_{\theta \geq 0} A_{p}^{\theta}$ and $A_{\infty}^{\infty}=\cup_{p \geq 1} A_{p}^{\infty}$. We remark that $A_{p}^{0}$ coincides with the Muckenhoupt's class of weights $A_{p}$ in [6] for all $1 \leq p<\infty$. However, in general, the class $A_{p}^{\infty}$ is strictly larger than the class $A_{p}$ for all $1 \leq p<\infty$. The following properties hold for the new classes $A_{p}^{\infty}$, see proposition 5 in [4].

Proposition 2.1 ([4]). The following statements hold:

(i) $A_{p}^{\infty} \subset A_{q}^{\infty}$ for $1 \leq p \leq q<\infty$. 
(ii) If $\omega \in A_{p}^{\infty}$, with $p>1$ then there exists $\varepsilon>0$ such that $\omega \in A_{p-\varepsilon}^{\infty}$. Consequently, $A_{p}^{\infty}=\cup_{q<p} A_{q}^{\infty}$.

(iii) If $\omega \in A_{p}^{\infty}$ with $p \geq 1$, then there exist positive numbers $\delta, l$ and $C$ so that for all cubes $Q$,

$$
\left(\frac{1}{|Q|} \int_{Q} \omega^{1+\delta}(x) d x\right)^{\frac{1}{1+\delta}} \leq C\left(\frac{1}{|Q|} \int_{Q} \omega(x) d x\right)(1+r)^{l} .
$$

Proposition 2.2 ([13]). The following statements hold:

(i) $\omega \in A_{p}^{\theta}$ if and only if $\omega^{\frac{-1}{p-1}} \in A_{p^{\prime}}^{\theta}$, where $\frac{1}{p}+\frac{1}{p^{\prime}}=1$.

(ii) If $\omega_{1}, \omega_{2} \in A_{p}^{\theta}, p \geq 1$, then $\omega_{1}^{\alpha} \omega_{2}^{1-\alpha} \in A_{p}^{\theta}$ for any $0<\alpha<1$.

(iii) If $\omega \in A_{p}^{\theta}$, for $1 \leq p<\infty$, then

$$
\frac{1}{\varphi_{\theta}(Q)|Q|} \int_{Q}|f(y)| d y \leq C\left(\frac{1}{\omega(5 Q)} \int_{Q}|f|^{p} \omega(y) d y\right)^{\frac{1}{p}} .
$$

In particular, Let $f=\chi_{E}$ for any measurable set $E \subset Q$,

$$
\frac{|E|}{\varphi_{\theta}(Q)|Q|} \leq C\left(\frac{\omega(E)}{\omega(5 Q)}\right)^{\frac{1}{p}}
$$

Let $1 \leq p_{1}, \ldots, p_{m}<\infty$. For $\vec{\omega}=\left(\omega_{1}, \ldots, \omega_{m}\right)$. Set $\nu_{\omega}=\prod_{j=1}^{m} \omega_{j}^{p / p_{j}}$. For $\theta \geq 0$, we say that $\vec{\omega}$ is in the class $A_{\vec{p}}^{\theta}$ if

$$
\left(\frac{1}{Q} \int_{Q} \nu_{\vec{\omega}}(x) d x\right)^{\frac{1}{p}} \prod_{j=1}^{m}\left(\frac{1}{|Q|} \int_{Q} \omega_{j}^{1-p_{j}^{\prime}}(x) d x\right)^{\frac{1}{p_{j}^{\prime}}} \leq C(1+r)^{m \theta}
$$

for all cubes $Q$. When $p_{j}=1,\left(\frac{1}{|Q|} \int_{Q} \omega_{j}^{1-p_{j}^{\prime}}(x) d x\right)^{\frac{1}{p_{j}^{\prime}}}$ is understood $\left(\inf _{x \in Q} \omega_{j}(x)\right)^{-1}$.

For $1 \leq p_{1}, \ldots, p_{m}<\infty$, we set $A_{\vec{p}}^{\infty}=\cup_{\theta \geq 0} A_{\vec{p}}^{\theta}$. When $\theta=0$, the class $A_{\vec{p}}^{0}$ coincides with the class of multiple weights $A_{\vec{p}}$ introduced by [8]. The following result gives a characterization of the class $A_{\vec{p}}^{\infty}$.

Proposition 2.3 ([2]). Let $1 \leq p_{1}, \ldots, p_{m}<\infty$ and $\vec{\omega}=\left(\omega_{1}, \ldots, \omega_{m}\right)$. Then the following statements are equivalent:

(i) $\vec{\omega} \in A_{\vec{p}}^{\infty}$;

(ii) $\omega_{j}^{1-p_{j}^{\prime}} \in A_{m p_{j}^{\prime}}^{\infty}, j=1, \ldots, m$ and $\nu_{\vec{\omega}} \in A_{m p}^{\infty}$.

The class $A_{\vec{p}}^{\infty}$ is not increasing which means that for $\vec{p}=\left(p_{1}, \ldots, p_{m}\right)$ and $\vec{q}=$ $\left(q_{1}, \ldots, q_{m}\right)$ with $p_{j} \leq q_{j}, j=1, \ldots, m$, the following may not be true $A_{\vec{p}}^{\infty} \subset A_{\vec{q}}^{\infty}$. However, we have the following result. 
Proposition 2.4 ([2]). Let $1 \leq p_{1}, \ldots, p_{m}<\infty$ and $\vec{\omega}=\left(\omega_{1}, \ldots, \omega_{m}\right) \in A_{\vec{p}}^{\infty}$. Then,

(i) For any $r \geq 1, \vec{\omega} \in A_{r \vec{p}}^{\infty}$;

(ii) If $1<p_{1}, \ldots, p_{m}<\infty$, then there exists $r>1$ so that $\vec{\omega} \in A_{\vec{p} / r}^{\infty}$.

\subsection{New BMO Function Spaces $B M O_{\infty}$}

In this section, we will recall the definition and some basic properties of the new $B M O$ function spaces. According to [3], the new BMO space $B M O_{\theta}$ with $\theta \geq 0$ is defined as a set of all locally integrable function $b$ satisfying

$$
\frac{1}{|Q|} \int_{Q}\left|b(y)-b_{B}\right| d y \leq C(1+r)^{\theta}
$$

where $Q=Q(x, r)$ and $b_{B}=\frac{1}{|Q|} \int_{Q} b$. A norm for $b \in B M O_{\theta}$, denoted by $\|b\|_{\theta}$, is given by the infimum of the constants satisfying (2.2). Clearly $B M O_{\theta_{1}} \subset B M O_{\theta_{2}}$ for $\theta_{1} \leq \theta_{2}$ and $B M O_{0}=B M O$. We define $B M O_{\infty}=\cup_{\theta \geq 0} B M O_{\theta}$.

The following result can be considered to be a variant of John-Nirenberg inequality for the spaces $B M O_{\infty}$.

Proposition 2.5 ([4]). Let $\theta>0, s \geq 1$. If $b \in B M O_{\theta}$ then for all $Q=Q(x, r)$

(i)

$$
\left(\frac{1}{|Q|} \int_{Q}\left|b(y)-b_{Q}\right|^{s} d x\right)^{\frac{1}{s}} \leq\|b\|_{\theta}(1+r)^{\theta}
$$

(ii)

$$
\left(\frac{1}{\left|3^{k} Q\right|} \int_{3^{k} Q}\left|b(y)-b_{B}\right|^{s} d x\right)^{\frac{1}{s}} \leq\|b\|_{\theta} k\left(1+3^{k} r\right)^{\theta},
$$

for all $k \in \mathbb{N}$.

Proposition 2.6 ([14]). Suppose that $f$ is in $B M O_{\theta}$, there exist positive constants $\gamma$ and $C$ such that

$$
\sup _{Q} \frac{1}{|Q|} \int_{Q} \exp \left\{\frac{\gamma\left|f(x)-f_{Q}\right|}{\|f\|_{B M O_{\theta}}(1+r)^{\theta}}\right\} d x \leq C
$$

\subsection{Orlicz Norms}

For $\Phi(t)=t\left(1+\log ^{+} t\right)$ and a cube $Q$ in $\mathbb{R}^{n}$ we will consider the average $\|f\|_{\Phi, Q}$ of a function $f$ given by the Luxemburg norm

$$
\|f\|_{\Phi, Q}=\inf \left\{\lambda>0: \frac{1}{|Q|} \int_{Q} \Phi\left(\frac{|f(x)|}{\lambda}\right) d x \leq 1\right\} .
$$

We will need the several basic estimates from the theory of Orlicz spaces. For more details about these spaces the reader may consult the book [15] or [16], we first recall that $\|f\|_{\Phi, Q}>1$ if and only if $\frac{1}{|Q|} \int_{Q} \Phi(|f(x)|) d x>1$. 
Next, we note that the generalized Hölder inequality in Orlicz spaces together with the John-Nirenberg inequality implies that

$$
\frac{1}{|Q|} \int_{Q}\left|\left(b(y)-b_{Q}\right) f(y)\right| d y \leq\|b\|_{B M O}\|f\|_{L(\log L), Q} .
$$

By proposition 2.6 and the generalized Hölder inequalitiy in Orlicz spaces, we get

$$
\frac{1}{|Q|} \int_{Q}\left|\left(b(y)-b_{Q}\right) f(y)\right| d y \leq\|b\|_{B M O_{\theta}}\|f\|_{L(\log L), Q}(1+r)^{\theta} .
$$

We will employ several times the following simple Kolmogorov inequality. Let $0<p<$ $q<\infty$, then there is a constant $C=C_{p, q}$ such that for any measurable function $f$, we have

$$
\|f\|_{L^{p}\left(Q, \frac{d x}{|Q|}\right)} \leq\|f\|_{L^{q}\left(Q, \frac{d x}{|Q|}\right)} .
$$

\subsection{Maximal Functions and Sharp Maximal Functions}

We now define a maximal operator $M_{\varphi, \eta}$ for $0<\eta<\infty$ by

$$
M_{\varphi, \eta} f(x)=\sup _{x \in Q} \frac{1}{\varphi(Q)^{\eta}|Q|} \int_{Q}|f(y)| d y .
$$

Proposition 2.7 ([13]). Let $1<p<\infty, p^{\prime}=\frac{p}{p-1}$ and suppose that $\omega \in A_{p}^{\eta}$ for $\eta>0$. There exists a constant $C>0$ such that

$$
\left\|M_{\varphi, p^{\prime} \eta} f\right\|_{L^{p}(\omega)} \leq C\|f\|_{L^{p}(\omega)} .
$$

Let $0<\eta<\infty$. As in [13], we define the dyadic maximal operator $M_{\varphi, \eta}^{\triangle}$ by

$$
M_{\varphi, \eta}^{\triangle} f(x)=\sup _{x \in Q(\text { dyadiccube })} \frac{1}{\varphi(Q)^{\eta}|Q|} \int_{Q}|f(x)| d x .
$$

And the dyadic sharp maximal operator $M_{\varphi, \eta}^{\sharp, \Delta}$ is defined by

$$
\begin{aligned}
M_{\varphi, \eta}^{\sharp, \Delta} f(x) & =\sup _{x \in Q, r<1} \frac{1}{|Q|} \int_{Q\left(x_{0}, r\right)}\left|f(y)-f_{Q}\right| d y+\sup _{x \in Q, r \geq 1} \frac{1}{\varphi(Q)^{\eta}|Q|} \int_{Q\left(x_{0}, r\right)}|f(y)| d y \\
& \simeq \sup _{x \in Q, r<1} \inf _{C} \frac{1}{|Q|} \int_{Q\left(x_{0}, r\right)}|f(y)-C| d y+\sup _{x \in Q, r \geq 1} \frac{1}{\varphi(Q)^{\eta}|Q|} \int_{Q\left(x_{0}, r\right)}|f(y)| d y,
\end{aligned}
$$

where $f_{Q}=\frac{1}{|Q|} \int_{Q} f(x) d x$ and $Q$ 's are dyadic cubes.

Proposition 2.8 ([13]). Let $1<p<\infty, \omega \in A_{p}^{\infty}, 0<\eta<\infty$ and $f \in L^{p}(\omega)$, then

$$
\|f\|_{L^{p}(\omega)} \leq\left\|M_{\varphi, \eta}^{\triangle} f\right\|_{L^{p}(\omega)} \leq C\left\|M_{\varphi, \eta}^{\sharp, \Delta} f\right\|_{L^{p}(\omega)} .
$$

We define a variant of dyadic maximal operator and dyadic sharp maximal operator as follows

$$
\begin{aligned}
& M_{\delta, \varphi, \eta}^{\Delta} f(x)=M_{\varphi, \eta}^{\triangle}\left(|f|^{\delta}\right)^{\frac{1}{\delta}}(x), \\
& M_{\delta, \varphi, \eta}^{\sharp, \triangle} f(x)=M_{\varphi, \eta}^{\sharp, \triangle}\left(|f|^{\delta}\right)^{\frac{1}{\delta}}(x) .
\end{aligned}
$$


Proposition 2.9 ([13]).Let $1<p<\infty, \omega \in A_{\infty}^{\infty}, 0<\eta<\infty$ and $\delta>0$. Let $\psi$ : $(0, \infty) \mapsto(0, \infty)$ be doubling, that is, $\psi(2 a) \leq \psi(a)$ for $a>0$. Then, there exists $a$ constant $C$ depending upon the $A_{\infty}^{\infty}$ condition of $\omega$ and doubling condition of $\psi$ such that

$$
\begin{gathered}
\sup _{\lambda>0} \psi(\lambda) \omega\left(\left\{y \in \mathbb{R}^{n}: M_{\delta, \varphi, \eta}^{\triangle} f(y)>\lambda\right\}\right) \leq C \sup _{\lambda>0} \psi(\lambda) \omega\left(\left\{y \in \mathbb{R}^{n}: M_{\delta, \varphi, \eta}^{\sharp, \Delta} f(y)>\lambda\right\}\right), \\
M_{L(\log L), \varphi, \eta} f(x)=\sup _{x \in Q} \frac{1}{\varphi(Q)^{\eta}}\|f\|_{L(\log L), Q} .
\end{gathered}
$$

Given $\vec{f}=\left(f_{1}, \ldots, f_{m}\right)$, we define the maximal operators $\mathbb{M}_{\varphi, \eta}$ and $\mathbb{M}_{L(\log L), \varphi, \eta}$ by

$$
\mathbb{M}_{\varphi, \eta}(\vec{f})(x)=\sup _{x \in Q} \prod_{j=1}^{m} \frac{1}{\varphi(Q)^{\eta}}\left\|f_{j}\right\|_{Q}
$$

and

$$
\mathbb{M}_{L(\log L), \varphi, \eta}(\vec{f})(x)=\sup _{x \in Q} \prod_{j=1}^{m} \frac{1}{\varphi(Q)^{\eta}}\left\|f_{j}\right\|_{L(\log L), Q}
$$

\section{Estimates for Multilinear Operators}

Theorem 3.1 Let $T$ is a multilinear operator satisfying (1.1)-(1.4) and let $0<\delta<\frac{1}{m}$ and $\eta>0$. Then for all $\vec{f}$ in any product of $L^{q_{j}}\left(\mathbb{R}^{n}\right)$ spaces, with $1 \leq q_{j}<\infty$, there exists $a$ constant $C$ such that

$$
M_{\delta, \varphi, \eta}^{\sharp, \Delta}(T(\vec{f}))(x) \leq C \mathbb{M}_{\varphi, \eta}(\vec{f})(x) .
$$

Proof We fix $x \in \mathbb{R}^{n}$ and Let $x \in Q=Q\left(x_{0}, r\right)$ (dyadic cube). To prove (3.1), we consider two cases about $r$.

Case 1. When $r \leq 1$. Since $0<\delta<\frac{1}{m}<1$, for any number $C$ we can estimate

$$
\left(\frac{1}{|Q|} \int_{Q}|T(\vec{f})(z)|^{\delta}-|C|^{\delta} \mid d z\right)^{\frac{1}{\delta}} \leq\left(\frac{1}{|Q|} \int_{Q}|T(\vec{f})(z)-C|^{\delta} d z\right)^{\frac{1}{\delta}}
$$

Let $Q^{*}=3 Q$, we split each $f_{i}$ as $f_{i}=f_{i}^{0}+f_{i}^{\infty}$ where $f_{i}^{0}=f \chi Q^{*}$ and $f_{i}^{\infty}=f_{i}-f_{i}^{0}$. This yields

$$
\prod_{j=1}^{m} f_{j}\left(y_{j}\right)=\sum_{\alpha_{1}, \ldots, \alpha_{m} \in\{0, \infty\}} f_{1}^{\alpha_{1}}\left(y_{1}\right) \ldots f_{m}^{\alpha_{m}}\left(y_{m}\right)=\prod_{j=0}^{m} f_{j}^{0}\left(y_{j}\right)+\sum^{\prime} f_{1}^{\alpha_{1}}\left(y_{1}\right) \ldots f_{m}^{\alpha_{m}}\left(y_{m}\right),
$$

where each term in $\sum^{\prime}$ contains at least one $\alpha_{j} \neq 0$.

Thus, Let $C=\sum^{\prime} C_{\alpha_{1}}, \cdots, C_{\alpha_{m}}$, we have

$$
\begin{aligned}
\left(\frac{1}{|Q|} \int_{Q}|T(\vec{f})(z)-C|^{\delta} d z\right)^{\frac{1}{\delta}} & \leq\left(\frac{1}{|Q|} \int_{Q}\left|T\left(f_{1}^{0}, \ldots, f_{m}^{0}\right)(z)\right|^{\delta} d z\right)^{\frac{1}{\delta}} \\
& +\sum^{\prime}\left(\frac{1}{|Q|} \int_{Q}\left|T\left(f_{1}^{\alpha_{1}}, \ldots, f_{m}^{\alpha_{m}}\right)(z)-C_{\alpha_{1}, \ldots, \alpha_{m}}\right|^{\delta} d z\right)^{\frac{1}{\delta}} \\
& :=I+I I .
\end{aligned}
$$


By Kolmogorov inequality, we have

$$
\begin{aligned}
I & \leq C\left\|T\left(f_{1}^{0}, \ldots, f_{m}^{0}\right)\right\|_{L^{\frac{1}{m}, \infty}\left(Q, \frac{d x}{|Q|}\right)} \leq C \prod_{j=1}^{m} \frac{1}{|3 Q|} \int_{3 Q}\left|f_{j}(z)\right| d z \\
& \leq C \prod_{j=1}^{m} \frac{1}{\varphi(3 Q)^{\eta}|3 Q|} \int_{3 Q}\left|f_{j}(z)\right| d z \leq C \mathbb{M}_{\varphi, \eta}(\vec{f})
\end{aligned},
$$

since $T: L^{1} \times \cdots \times L^{1} \rightarrow L^{\frac{1}{m}}$.

We set now $C_{\alpha_{1}}, \cdots, \alpha_{m}=T\left(f_{1}^{\alpha_{1}}, \cdots, f_{m}^{\alpha_{m}}\right)(x)$, for any $z \in Q$, we will show the following estimate:

$$
\sum^{\prime}\left|T\left(f_{1}^{\alpha_{1}}, \cdots, f_{m}^{\alpha_{m}}\right)(z)-T\left(f_{1}^{\alpha_{1}}, \cdots, f_{m}^{\alpha_{m}}\right)(x)\right| \leq C \mathbb{M}_{\varphi, \eta}(\vec{f}(x)) .
$$

Consider first the case when $\alpha_{1}=\cdots=\alpha_{m}=\infty$ and define $T\left(\vec{f}^{\infty}\right)=T\left(f_{1}^{\infty}, \cdots, f_{m}^{\infty}\right)$.

$$
\begin{aligned}
& \left(\frac{1}{|Q|} \int_{Q}\left|T\left(f_{1}^{\infty}, \cdots, f_{m}^{\infty}\right)(z)-T\left(f_{1}^{\infty}, \cdots, f_{m}^{\infty}\right)(x)\right|^{\delta} d z\right)^{\frac{1}{\delta}} \\
& \quad \leq C\left(\frac{1}{|Q|} \int_{Q}\left|T\left(f_{1}^{\infty}, \cdots, f_{m}^{\infty}\right)(z)-T\left(f_{1}^{\infty}, \cdots, f_{m}^{\infty}\right)(x)\right| d z\right) \\
& \quad \leq C \frac{1}{|Q|} \int_{Q} \int_{\left(\mathbb{R}^{n} \backslash 3 Q\right)^{m}} \frac{|x-z|^{\varepsilon}}{\left(\left|z-y_{1}\right|+\cdots+\left|z-y_{m}\right|\right)^{n m+\varepsilon}} \\
& \quad \leq C \int_{Q} \sum_{k=1}^{\infty} \frac{1}{\prod_{j=1}^{m}\left|f_{j}\left(y_{j}\right)\right|} \\
& \quad \leq C \sum_{k=1}^{\infty} \frac{3^{-k \varepsilon}}{\varphi\left(2^{k+1} Q\right)^{N}} \int_{\left(3^{k+1} Q\right)^{m} \backslash\left(3^{k} Q\right)^{m}} \prod_{j=1}^{m}\left|f_{j=1}^{m}\left(y_{j}\right)\right| d \vec{y} d z
\end{aligned}
$$

if taking $N \geq m \eta$.

We are now to consider $\alpha_{j_{1}}=\cdots=\alpha_{j_{l}}=0$ for some $\left\{j_{1}, \cdots, j_{l}\right\} \subset\{1, \cdots, m\}$ and $1 \leq l<m$.

$$
\begin{aligned}
& \left(\frac{1}{|Q|} \int_{Q}\left|T\left(f_{1}^{\alpha_{1}}, \cdots, f_{m}^{\alpha_{m}}\right)(z)-T\left(f_{1}^{\alpha_{1}}, \cdots, f_{m}^{\alpha_{m}}\right)(x)\right|^{\delta} d z\right)^{\frac{1}{\delta}} \\
& \leq C \frac{1}{|Q|} \int_{Q}\left|T\left(f_{1}^{\alpha_{1}}, \cdots, f_{m}^{\alpha_{m}}\right)(z)-T\left(f_{1}^{\alpha_{1}}, \cdots, f_{m}^{\alpha_{m}}\right)(x)\right| d z \\
& \leq C \frac{1}{|Q|} \int_{Q} \prod_{j \in\left\{j_{1}, \cdots, j_{l}\right\}} \int_{3 Q}\left|f_{j}\left(y_{j}\right)\right| d y_{j} \\
& \times \int_{\left(\mathbb{R}^{n} \backslash 3 Q\right)^{m-l}} \frac{|x-z|^{\varepsilon} \prod_{j \notin\left\{j_{1}, \cdots, j_{l}\right\}}\left|f_{j}\left(y_{j}\right)\right| d y_{j}}{\left(\left|z-y_{1}\right|+\cdots+\left|z-y_{m}\right|\right)^{n m+\varepsilon}\left(1+\left|z-y_{1}\right|+\cdots+\left|z-y_{m}\right|\right)^{N}} d z \\
& \leq C \prod_{j \in\left\{j_{1}, \cdots, j_{l}\right\}} \int_{3 Q}\left|f_{j}\left(y_{j}\right)\right| d y_{j} \\
& \times \sum_{k=1}^{\infty} \frac{|Q|^{\varepsilon / n}}{\left(3^{k}|Q|^{\frac{1}{n}}\right)^{n m+\varepsilon} \varphi\left(2^{k+1} Q\right)^{N}} \int_{\left(3^{k+1} Q\right)^{m-l}} \prod_{j \notin\left\{j_{1}, \cdots, j_{l}\right\}}\left|f_{j}\left(y_{j}\right)\right| d y_{j} \\
& \leq C \sum_{k=1}^{\infty} \frac{|Q|^{\varepsilon / n}}{\left(3^{k}|Q|^{1 / n}\right)^{n m+\varepsilon} \varphi\left(2^{k+1} Q\right)^{N}} \int_{\left(3^{k+1} Q\right)^{m}} \prod_{j=1}^{m}\left|f_{j}\left(y_{j}\right)\right| d \vec{y} \\
& \leq C \sum_{k=1}^{\infty} \frac{k}{3^{k \varepsilon} \varphi\left(2^{k+1} Q\right)^{N}} \prod_{j=1}^{m}\left|f_{j}\right|_{3^{k+1}} Q \leq C \mathbb{M}_{\varphi, \eta} \vec{f}(x),
\end{aligned}
$$


if taking $N \geq m \eta$.

Case 2. When $r \geq 1$, since $0<\delta<\frac{1}{m}<1$, and $\eta>0$, by Kolmogorov' s inequality and $T: L^{1} \times \cdots \times L^{1} \rightarrow L^{1 / m}$, we have

$$
\begin{aligned}
& \left(\frac{1}{\varphi(Q)^{\eta}|Q|} \int_{Q}|T(\vec{f})(x)|^{\delta} d x\right)^{\frac{1}{\delta}} \\
& \leq C \frac{1}{\varphi(Q)^{\eta / \delta}}\left(\frac{1}{|Q|} \int_{Q}\left|T\left(f_{1}^{0}, \cdots, f_{m}^{0}\right)(x)\right|^{\delta} d x\right)^{\frac{1}{\delta}} \\
& +\sum^{\prime} \frac{1}{\varphi(Q)^{\eta / \delta}}\left(\frac{1}{|Q|} \int_{Q}\left|T\left(f_{1}^{\alpha_{1}}, \cdots, f_{m}^{\alpha_{m}}\right)(z)\right|^{\delta}\right)^{\frac{1}{\delta}} \\
& \leq C \frac{1}{\varphi(Q)^{\eta m}}\left\|T\left(f_{1}^{0}, \cdots \cdot, f_{m}^{0}\right)\right\|_{L^{\frac{1}{m}, \infty}\left(Q, \frac{d x}{|Q|}\right)} \\
& +\sum^{\prime} \frac{1}{\varphi(Q)^{\eta / \delta}}\left(\frac{1}{|Q|} \int_{Q}\left|T\left(f_{1}^{\alpha_{1}}, \cdots, f_{m}^{\alpha_{m}}\right)(z)\right|^{\delta}\right)^{\frac{1}{\delta}} \\
& \leq C \frac{1}{\varphi(Q)^{\eta m}} \prod_{j=1}^{m} \frac{1}{|3 Q|} \int_{3 Q}\left|f_{j}(z)\right| d z+\sum^{\prime} \frac{1}{\varphi(Q)^{\eta / \delta}}\left(\frac{1}{|Q|} \int_{Q}\left|T\left(f_{1}^{\alpha_{1}}, \cdots, f_{m}^{\alpha_{m}}\right)(z)\right|^{\delta}\right)^{\frac{1}{\delta}} \\
& \leq C \mathbb{M}_{\varphi, \eta}(\vec{f})(x)+\sum^{\prime} \frac{1}{\varphi(Q)^{\eta / \delta}}\left(\frac{1}{|Q|} \int_{Q}\left|T\left(f_{1}^{\alpha_{1}}, \cdots, f_{m}^{\alpha_{m}}\right)(z)\right|^{\delta}\right)^{\frac{1}{\delta}} .
\end{aligned}
$$

Consider now $\alpha_{1}=\cdots=\alpha_{m}=\infty$, taking $N=m \eta$, we obtain that

$$
\begin{aligned}
\frac{1}{\varphi_{\eta}(Q)^{1 / \delta}} & \left(\frac{1}{|Q|} \int_{Q}\left|T\left(f_{1}^{\infty}, \cdots, f_{m}^{\infty}\right)(z)\right|^{\delta}\right)^{\frac{1}{\delta}} \leq C \frac{1}{|Q|} \int_{Q}\left|T\left(f_{1}^{\infty}, \cdots, f_{m}^{\infty}\right)(z)\right| d z \\
& \leq C \frac{1}{|Q|} \int_{Q} \int_{\left(\mathbb{R}^{n} \backslash 3 Q\right)^{m}} \frac{\prod_{j=1}^{m}\left|f_{j}\left(y_{j}\right)\right|}{\left(\left|z-y_{1}\right|+\cdots+\left|z-y_{m}\right|\right)^{n m+N}} d \vec{y} d z \\
& \leq C \frac{1}{|Q|} \int_{Q} \sum_{k=1}^{\infty} \int_{\left(3^{k+1} Q\right)^{m} \backslash\left(3^{k} Q\right)^{m}} \frac{\prod_{j=1}^{m}\left|f_{j}\left(y_{j}\right)\right|}{\left(\left|z-y_{1}\right|+\cdots+\left|z-y_{m}\right|\right)^{n m+N}} d \vec{y} d z \\
& \leq C \sum_{k=1}^{\infty} \frac{1}{\left(3^{k}|Q|^{\frac{1}{n}}\right)^{N}} \frac{1}{\left(3^{k}|Q|^{\frac{1}{n}}\right)^{n m}} \int_{\left(3^{k+1} Q\right)^{m}} \prod_{j=1}^{m}\left|f_{j}\left(y_{j}\right)\right| d y_{1} \cdots d y_{m} \\
& \leq C \sum_{k=1}^{\infty} 3^{-k} \frac{1}{\varphi\left(3^{k+1} Q\right)^{\eta m}} \prod_{j=1}^{m}\left|f_{j}\right|_{3^{k+1} Q} \leq C \mathbb{M}_{\varphi, \eta}(\vec{f})(x) .
\end{aligned}
$$

Next we consider the case $\alpha_{j_{1}}=\alpha_{j_{2}}=\cdots=\alpha_{j_{l}}=0$ for some $\left\{j_{1}, \cdots, j_{l}\right\} \subset\{1, \cdots, m\}$, where $1 \leq l<m$ and take $N=m \eta+1$, then

$$
\begin{aligned}
& \frac{1}{\varphi_{\eta}(Q)^{1 / \delta}}\left(\frac{1}{|Q|} \int_{Q}\left|T\left(f_{1}^{\alpha_{1}}, \cdots, f_{m}^{\alpha_{m}}\right)(z)\right|^{\delta}\right)^{\frac{1}{\delta}} \\
& \quad \leq \frac{C}{|Q|} \int_{Q} \prod_{j \in\left\{j_{1}, \cdots, j_{l}\right\}} \int_{3 Q}\left|f_{j}\left(y_{j}\right)\right| d y_{j} \int_{\left(\mathbb{R}^{n} \backslash 3 Q\right)^{m-l}} \frac{\prod_{j \notin\left\{j_{1}, \cdots, j_{l}\right\}}\left|f_{j}\left(y_{j}\right)\right| d y_{j}}{\left(\left|z-y_{1}\right|+\cdots+\left|z-y_{m}\right|\right)^{N+n m}} d z \\
& \quad \leq C \prod_{j \in\left\{j_{1}, \cdots, j_{l}\right\}} \int_{3 Q}\left|f_{j}\left(y_{j}\right)\right| d y_{j} \sum_{k=1}^{\infty} \int_{\left(3^{k+1} Q\right)^{m-l} \backslash\left(3^{k} Q\right)^{m-l}} \frac{\prod_{j \notin\left\{j_{1}, \cdots, j_{l}\right\}}\left|f_{j}\left(y_{j}\right)\right| d y_{j}}{\left(3^{k}|Q|^{\frac{1}{n}}\right)^{n m+N}} \\
& \quad \leq C \sum_{k=1}^{\infty} 3^{-k} \frac{1}{\varphi\left(3^{k+1} Q\right)^{\eta m}} \prod_{j=1}^{m}\left|f_{j}\right|_{3^{k+1} Q} \leq C \mathbb{M}_{\varphi, \eta}(\vec{f})(x) .
\end{aligned}
$$

The proof is complete. 
Theorem 3.2 Let $1 \leq p_{j}<\infty, j=1, \cdots, m$, and $\frac{1}{p}=\frac{1}{p_{1}}+\cdots+\frac{1}{p_{m}}$. Let $v, \omega_{j} \in A_{p}^{\theta}$ for some $\theta>0$. Then the inequality

$$
\left\|\mathbb{M}_{\varphi, \theta}(\vec{f})\right\|_{L^{p, \infty}(v)} \leq C \prod_{j=1}^{m}\left\|f_{j}\right\|_{L^{p_{j}}\left(\omega_{j}\right)}
$$

holds for any $\vec{f}$ if and only if

$$
\sup _{Q}\left(\frac{1}{\varphi(Q)^{\theta}|Q|} \int_{Q} v\right)^{\frac{1}{p}} \prod_{j=1}^{m}\left(\frac{1}{\varphi(Q)^{\theta}|Q|} \int_{Q} \omega_{j}^{1-p_{j}^{\prime}}\right)^{\frac{1}{p_{j}^{\prime}}}<\infty
$$

where $\left(\frac{1}{\varphi(Q)^{\theta}|Q|} \int_{Q} \omega_{j}^{1-p_{j}^{\prime}}\right)^{\frac{1}{p_{j}^{\prime}}}$ in the case $p_{j}=1$ is understood as $\left(\inf _{Q} \omega_{j}\right)^{-1}$.

The proof of Theorem 3.2 is almost the same as the proof of Theorem 3.3 in [8], so we omit the details here.

Theorem 3.3 Let $1<p_{j}<\infty, j=1, \cdots, m$ and $\frac{1}{p}=\frac{1}{p_{1}}+\cdots+\frac{1}{p_{m}}$. Let $\vec{\omega} \in A_{\vec{p}}^{\infty}$, then there exists some $\eta_{0}>0$ depending on $p, m, p_{j}$ and the condition of $\vec{\omega} \in A_{\vec{p}}^{\infty}$ such that the inequality $\left\|\mathbb{M}_{\varphi, \eta_{0}}(\vec{f})\right\|_{L^{p}\left(v_{\vec{\omega}}\right)} \leq C \prod_{j=1}^{m}\left\|f_{j}\right\|_{L^{p_{j}\left(\omega_{j}\right)}}$ holds for every $\vec{f}$.

Proof Since $\vec{\omega} \in A_{\vec{p}}^{\infty}$, then there exists $\theta_{1}>0$ such that $\vec{\omega} \in A_{\vec{p}}^{\theta_{1}}$. By proposition 2.3 each $\omega_{j}^{1-p_{j}^{\prime}} \in A_{m p_{j}^{\prime}}^{\theta_{2}}$ and $\nu_{\omega} \in A_{p m}^{\theta_{2}}$. By proposition 2.1(iii), there exist $t_{j}>1, l>0$ and $c>0$ such that for all $1 \leq t \leq t_{j}$ and for any cube $Q$,

$$
\left(\frac{1}{|Q|} \int_{Q} \omega_{j}^{\frac{-t}{p_{j}-1}}\right)^{\frac{1}{t}} \leq C\left(\frac{1}{|Q|} \int_{Q} \omega_{j}^{\frac{-1}{p_{j}-1}}\right)(1+r)^{l},
$$

where $r$ is the length of the cube $Q$.

Let $\xi=\min _{1 \leq j \leq m} r_{j}$ and $q=\max _{1 \leq j \leq m} \frac{p m}{p m+\left(1-\frac{1}{\xi}\right)\left(p_{j}-1\right)}$, and note that $q p_{j}>$ 1 for any $j$.

By Hölder's inequality, we obtain that

$$
\frac{1}{|Q|} \int_{Q}\left|f_{j}\right| \leq \frac{1}{|Q|}\left(\int_{Q}\left(\left|f_{j}\right|^{p_{j}} \omega_{j} / \nu_{\vec{\omega}}\right)^{q} \cdot v_{\vec{\omega}}\right)^{\frac{1}{q p_{j}}}\left(\int_{Q}\left(\omega_{j}^{q} v_{\vec{\omega}}^{1-q}\right)^{\frac{-1}{q p_{j}-1}}\right)^{1-\frac{1}{q p_{j}}}
$$

Let $\gamma_{j}=\frac{q p_{j}-1}{(1-q)(p m-1)}$. By the definition of $q, \gamma_{j}>1$ for any $j$, and using Hölder's inequality, we get

$$
\int_{Q}\left(\omega_{j}^{q} \nu_{\vec{\omega}}^{1-q}\right)^{\frac{-1}{q p_{j}-1}} \leq\left(\int_{Q} \omega_{j}^{\frac{-q \gamma_{j}^{\prime}}{q p_{j}-1}}\right)^{\frac{1}{\gamma_{j}^{\prime}}}\left(\int_{Q} \nu_{\vec{\omega}}^{\frac{-1}{p m-1}}\right)^{\frac{1}{\gamma_{j}}} .
$$

Note that for any $j$,

$$
\frac{q\left(p_{j}-1\right) \gamma_{j}^{\prime}}{q p_{j}-1}=\frac{q\left(p_{j}-1\right)}{q\left(p_{j}-1\right)-(1-q) p m} \leq \xi
$$


Thus, by (3.2) we obtain that

$$
\begin{aligned}
\int_{Q} \omega_{j}^{\frac{-q \gamma_{j}^{\prime}}{q p_{j}-1}} & =\int_{Q} \omega_{j}^{\frac{-1}{p_{j}-1} \frac{q\left(p_{j}-1\right) \gamma_{j}^{\prime}}{q p_{j}-1}} \\
& \leq C|Q|^{1-\frac{q\left(p_{j}-1\right) \gamma_{j}^{\prime}}{q p_{j}-1}}\left(\int_{Q} \omega_{j}^{\frac{-1}{p_{j}-1}}\right)^{\frac{q\left(p_{j}-1\right) \gamma_{j}^{\prime}}{q p_{j}-1}}(1+r)^{\frac{q\left(p_{j}-1\right) \gamma_{j}^{\prime} l}{q p_{j}-1}} .
\end{aligned}
$$

Using (3.4) and (3.5) and $\nu_{\omega} \in A_{p m}^{\theta_{2}}$, set $\theta^{\prime}=\left(\theta_{2} m p(1-q) l\right) /\left(q p_{j} p_{j}^{\prime}\right)$, we obtain

$$
\begin{aligned}
& \left(\int_{Q}\left(\omega_{j}^{q} v_{\vec{\omega}}^{1-q}\right)^{\frac{-1}{q p_{j}-1}}\right)^{1-\frac{1}{q p_{j}}} \\
& \quad \leq C|Q|^{\frac{-p m(1-q)}{q p_{j}}}\left(\int_{Q} \omega_{j}^{\frac{-1}{p_{j}-1}}\right)^{1-\frac{1}{p_{j}}}(1+r)^{l\left(1-\frac{1}{p_{j}}\right)}\left(\int_{Q} \nu_{\vec{\omega}}^{\frac{-1}{p m-1}}\right)^{\frac{(1-q)(p m-1)}{q p_{j}}} \\
& \quad \leq C \frac{1}{v_{\omega}(Q)^{\frac{1-q}{q p_{j}}}}\left(\int_{Q} \omega_{j}^{\frac{-1}{p_{j}-1}}\right)^{1-\frac{1}{p_{j}}}(1+r)^{\theta^{\prime}} .
\end{aligned}
$$

Combing this inequality with (3.3), Proposition2.2 (iii) and $\vec{\omega} \in A_{\vec{p}}^{\theta_{1}}$, we obtain

$$
\begin{aligned}
\frac{1}{(1+r)^{\left(\theta_{1}+\theta^{\prime}\right) m}} & \prod_{j=1}^{m} \frac{1}{|Q|} \int_{Q}\left|f_{j}\right| \\
& \leq C \prod_{j=1}^{m}\left(\frac{1}{v_{\vec{\omega}}(Q)} \int_{Q}\left(\left|f_{j}\right|^{p_{j}} \omega_{j} / \nu_{\vec{\omega}}\right)^{q} \nu_{\vec{\omega}}\right)^{\frac{1}{q p_{j}}} \\
& \left.\leq C \prod_{j=1}^{m}\left(\frac{1}{v_{\vec{\omega}}(5 Q)} \frac{\nu_{\vec{\omega}}(5 Q)}{v_{\vec{\omega}}(Q)} \int_{Q}\left(\left|f_{j}\right|^{p_{j}} \omega_{j} / \nu_{\vec{\omega}}\right)^{q} v_{\vec{\omega}}\right)\right)^{\frac{1}{q p_{j}}} \\
& \leq C(1+r)^{\frac{\theta_{2} m}{q}} \prod_{j=1}^{m}\left(\frac{1}{\nu_{\vec{\omega}}(5 Q)} \int_{Q}\left(\left|f_{j}\right|^{p_{j}} \omega_{j} / \nu_{\vec{\omega}}\right)^{q} \nu_{\vec{\omega}}\right)^{\frac{1}{q p_{j}}} .
\end{aligned}
$$

Therefore, we have

$$
\frac{1}{(1+r)^{\left(\theta_{1}+\theta^{\prime}+\theta_{2} / q\right) m}} \prod_{j=1}^{m} \frac{1}{|Q|} \int_{Q}\left|f_{j}\right| \leq C \prod_{j=1}^{m}\left(\frac{1}{\nu_{\vec{\omega}}(5 Q)} \int_{Q}\left(\left|f_{j}\right|^{p_{j}} \omega_{j} / \nu_{\vec{\omega}}\right)^{q} \nu_{\vec{\omega}}\right)^{\frac{1}{q p_{j}}}
$$

Let $\theta_{0}=\theta_{1}+\theta^{\prime}+\theta_{2} / q$ and $\eta_{0} \geq \theta_{0}$, we have

$$
\mathbb{M}_{\varphi, \eta_{0}} \vec{f}(x) \leq C \prod_{j=1}^{m}\left(M_{\nu_{\vec{\omega}}}\left(\left(\left|f_{j}\right|^{p_{j}} \omega_{j} / \nu_{\vec{\omega}}\right)^{q}\right)\right)^{\frac{1}{q P_{j}}}
$$

By Hölder's inequality and Proposition 2.7, the theorem is proved.

Corollary 3.1 Let $T$ be a multilinear operator satisfying (1.1)-(1.4). Suppose $\omega \in A_{\infty}^{\infty}$, $\eta>0$ and $p>0$. There exists $C>0$ depending on the $A_{\infty}^{\infty}$ constant of $\omega$ so that the inequalities

$$
\|T(\vec{f})\|_{L^{p}(\omega)} \leq C\left\|\mathbb{M}_{\varphi, \eta}(\vec{f})\right\|_{L^{p}(\omega)}
$$


and

$$
\|T(\vec{f})\|_{L^{p, \infty}(\omega)} \leq C\left\|\mathbb{M}_{\varphi, \eta}(\vec{f})\right\|_{L^{p, \infty}(\omega)}
$$

hold for all bounded functions $\vec{f}$ with compact support.

Proof It is enough to prove (3.6) when the right-hand side is finite (or there is nothing to prove). Applying Proposition 2.8 and Theorem 3.1, we have

$$
\|T(\vec{f})\|_{L^{p}(\omega)} \leq\left\|M_{\delta, \varphi, \eta}^{\Delta}(T(\vec{f}))\right\|_{L^{p}(\omega)} \leq C\left\|M_{\delta, \varphi, \eta}^{\sharp, \Delta}(T(\vec{f}))\right\|_{L^{p}(\omega)} \leq C\left\|\mathbb{M}_{\varphi, \eta}(\vec{f})\right\|_{L^{p}(\omega)} .
$$

Using similar arguments, we can obtain the weak-type estimate (3.7). The proof is complete.

Applying Proposition 2.3, Theorem 3.2, Theorem 3.3 and Corollary 3.1 above we get the following estimates, which has been proved by Bui in [2].

Corollary 3.2 Suppose $T$ is a multilinear operator satisfying (1.1)-(1.4), $\vec{w} \in A_{\vec{p}}^{\infty}$ and $\frac{1}{p}=\frac{1}{p_{1}}+\cdots+\frac{1}{p_{m}}$. we have

(i) If $1<p_{j}<\infty, j=1, \cdots, m$, then

$$
\|T(\vec{f})\|_{L^{p}\left(v_{\vec{\omega}}\right)} \leq C \prod_{j=1}^{m}\left\|f_{j}\right\|_{L^{p_{j}\left(\omega_{j}\right)}} .
$$

(ii) If $1 \leq p_{j}<\infty, j=1, \cdots, m$, and at least one of $p_{j}=1$, then

$$
\|T(\vec{f})\|_{L^{p, \infty}\left(v_{\vec{\omega}}\right)} \leq C \prod_{j=1}^{m}\left\|f_{j}\right\|_{L^{p_{j}\left(\omega_{j}\right)}} .
$$

\section{Estimates for Multilinear Commutators}

We first give a pointwise estimate using sharp maximal functions. To state the pointwise result we need to induce some additional for $m$-linear commutators involving $j B M O_{\theta}$ functions with $j \leq m$. As in [11], for positive integers $m$ and $j$ with $1 \leq j \leq m$, we denote by $C_{j}^{m}$ the family of all finite subsets $\sigma=\{\sigma(1), \cdots, \sigma(j)\}$ of $\{1, \cdots, m\}$ of $j$ different elements, where we always take $\sigma(k)<\sigma(l)$ if $k<l$. For any $\sigma \in C_{j}^{m}$, we associate the complementary sequence $\alpha^{\prime} \in C_{m-j}^{m}$ given by $\sigma^{\prime}=\{1, \cdots, m\} \backslash \sigma$ with the convention $C_{0}^{m}=\emptyset$. Given an $m$-tuple of functions $\vec{b}$ and $\sigma \in C_{j}^{m}$, we also use the notation $\vec{b}_{\sigma}$ for the $j$-tuple obtained from $\vec{b}$ given by $\left(b_{\sigma(1)}, \cdots, b_{\sigma(j)}\right)$.

Similarly to (1.6), we define for a multilinear operator $T, \sigma \in C_{j}^{m}$, and $\vec{b}=$ $\left(b_{\sigma(1)}, \cdots, b_{\sigma(j)}\right)$ in $B M O_{\vec{\theta}}^{j}$, the iterated commutator

$$
T_{\Pi \overrightarrow{b_{\sigma}}}\left(f_{1}, \ldots, f_{m}\right)=\left[b_{\sigma(1)},\left[b_{\sigma(2)}, \ldots\left[b_{\sigma(j-1)},\left[b_{\sigma(j)}, T\right]_{\sigma(j)}\right]_{\sigma(j-1} \ldots\right]_{\sigma(2)}\right]_{\sigma(1)}(\vec{f}) .
$$

That is, formally

$$
T_{\Pi \overrightarrow{b_{\sigma}}}(\vec{f})(x)=\int_{\mathbb{R}^{n m}} \prod_{i=1}^{j}\left(b_{\sigma(i)}(x)-b_{\sigma(i)}\left(y_{\sigma(i)}\right)\right) K\left(x, y_{1}, \ldots, y_{m}\right) f_{1}\left(y_{1}\right) \ldots f_{m}\left(y_{m}\right) d y_{1} \ldots d y_{m}
$$


Clearly $T_{\Pi \overrightarrow{b_{\sigma}}}=T_{\Pi \vec{b}}$ as defined before when $\sigma=\{1,2, \cdot, m\}$, while $T_{\Pi \overrightarrow{b_{\sigma}}}=T_{b_{j}}^{j}$ when $\sigma=\{j\}$. The pointwise estimate that will serve our purposes is the following.

Proposition 4.1 Let $T_{\Pi \vec{b}}$ be a multilinear commutator with $\vec{b} \in B M O_{\vec{\theta}}^{m}(\vec{\theta}=$ $\left(\theta_{1}, \cdots, \theta_{m}\right)$ and $T$ is a multilinear operator satisfying (1.1)-(1.4). Let $0<\delta<\varepsilon<1 / m$ and $\eta \geq\left(\theta_{1}+\cdots+\theta_{m}\right) /(1 / \delta-1 / \varepsilon)$. Then

$$
\begin{aligned}
M_{\delta, \varphi, \eta}^{\sharp, \Delta}\left(T_{\Pi \vec{b}}(\vec{f})\right)(x) \leq C & \prod_{j=1}^{m}\left\|b_{j}\right\|_{B M O_{\theta_{j}}}\left(\mathbb{M}_{L(\log L), \varphi, \eta}(\vec{f})(x)+M_{\varepsilon, \varphi, \eta}^{\Delta}(T(\vec{f}))(x)\right) \\
& +C \sum_{j=1}^{m-1} \sum_{\sigma \in C_{j}^{m}} \prod_{i=1}^{j}\left\|b_{\sigma(i)}\right\|_{B M O_{\sigma(i)}} M_{\varepsilon}\left(T_{\Pi \overrightarrow{b_{\sigma^{\prime}}}}(\vec{f})\right)(x)
\end{aligned}
$$

for all m-tuples $\vec{f}=\left(f_{1}, \cdots, f_{m}\right)$ of bounded measurable functions with compact support.

Proof We only prove the case $m=2$ and $\theta_{1}=\theta_{2}=\theta$ for simplicity. In the general case, there is no different computations but only more complicated. Fix then $b_{1}, b_{2} \in B M O_{\theta}$ and for any constants $\lambda_{1}$ and $\lambda_{2}$,

$$
\begin{aligned}
T_{\Pi \vec{b}}(\vec{f})(x)= & \left(b_{1}(x)-\lambda_{1}\right)\left(b_{2}(x)-\lambda_{2}\right) T\left(f_{1}, f_{2}\right)(x)-\left(b_{1}(x)-\lambda_{1}\right) T\left(f_{1},\left(b_{2}-\lambda_{2}\right) f_{2}\right) \\
& -\left(b_{2}(x)-\lambda_{2}\right) T\left(\left(b_{1}-\lambda_{1}\right) f_{1}, f_{2}\right)+T\left(\left(b_{1}-\lambda_{1}\right) f_{1},\left(b_{2}-\lambda_{2}\right) f_{2}\right)(x) .
\end{aligned}
$$

If we fix $x \in \mathbb{R}^{n}$, a dyadic cube $Q \ni x$ and any constants $c_{1}, c_{2}, c_{3}$, then since $0<\delta<\frac{1}{2}$, we can estimate (4.1) in two cases.

Case 1: $r \leq 1$. We have

$$
\begin{aligned}
&\left(\left.\frac{1}{|Q|} \int_{Q}|| T_{\Pi \vec{b}}(\vec{f})(z)\right|^{\delta}-\left|\sum_{j=1}^{3} c_{j}\right|^{\delta} \mid d z\right)^{\frac{1}{\delta}} \\
& \leq\left(\frac{1}{|Q|} \int_{Q}\left|T_{\Pi \vec{b}}(\vec{f})(z)-\sum_{j=1}^{3} c_{j}\right| d z\right)^{\frac{1}{\delta}} \\
& \leq C\left(\frac{1}{|Q|} \int_{Q}\left|\left(b_{1}(z)-\lambda_{1}\right)\left(b_{2}(z)-\lambda_{2}\right) T\left(f_{1}, f_{2}\right)(z)\right|^{\delta} d z\right)^{\frac{1}{\delta}} \\
&+C\left(\frac{1}{|Q|} \int_{Q}\left|\left(b_{1}(z)-\lambda_{1}\right) T\left(f_{1},\left(b_{2}-\lambda_{2}\right) f_{2}\right)(z)-c_{1}\right|^{\delta} d z\right)^{\frac{1}{\delta}} \\
&+C\left(\frac{1}{|Q|} \int_{Q}\left|\left(b_{2}(z)-\lambda_{2}\right) T\left(\left(b_{1}-\lambda_{1}\right) f_{1}, f_{2}\right)(z)-c_{2}\right|^{\delta} d z\right)^{\frac{1}{\delta}} \\
&+C\left(\frac{1}{|Q|} \int_{Q}\left|T\left(\left(b_{1}-\lambda_{1}\right) f_{1},\left(b_{2}(z)-\lambda_{2}\right) f_{2}\right)(z)-c_{3}\right|^{\delta} d z\right)^{\frac{1}{\delta}} \\
&:= I+I I+I I+I V .
\end{aligned}
$$


By selecting appropriate constants we estimate each term separately. Let $Q^{*}=3 Q$ and $\lambda_{j}=\left(b_{j}\right)_{Q^{*}}$ be the average of $b_{j}$ on $Q^{*}, j=1,2$. For any $1<q_{1}, q_{2}, q_{3}<\infty$ with $1=\frac{1}{q_{1}}+\frac{1}{q_{2}}+\frac{1}{q_{3}}$. And choosing $\delta$ sufficiently small so that $\delta q_{i}<1, i=1,2$ and $q_{3}<\varepsilon / \delta$.

By Proposition 2.5 and Hölder's inequalities, we have

$$
\begin{aligned}
I \leq & \left.C\left(\frac{1}{|Q|} \int_{Q}\left|b_{1}(z)-\lambda_{1}\right|^{\delta q_{1}} d z\right)^{\frac{1}{\delta q_{1}}}\right)\left(\frac{1}{|Q|} \int_{Q}\left|b_{2}(z)-\lambda_{2}\right|^{\delta q_{2}} d z\right)^{\frac{1}{\delta q_{2}}} \\
& \times\left(\frac{1}{|Q|} \int_{Q}\left|T\left(f_{1}, f_{2}\right)(z)\right|^{\delta q_{3}} d z\right)^{\frac{1}{\delta q_{3}}} \\
\leq & C\left\|b_{1}\right\|_{B M O_{\theta}}\left\|b_{2}\right\|_{B M O_{\theta}} M_{\varepsilon, \varphi, \eta}^{\triangle}\left(T\left(f_{1}, f_{2}\right)\right)(x) .
\end{aligned}
$$

Since II and III are symmetric, we only estimate II. we split each $f_{i}$ as $f_{i}=f_{i}^{0}+f_{i}^{\infty}$ where $f_{i}^{0}=f_{i} \chi Q^{*}$ and $f_{i}^{\infty}=f_{i}-f_{i}^{0}$. Let $c_{1}=\left(b_{1}(z)-\lambda_{1}\right) \sum_{j=1}^{3} c_{1 j}$, where $c_{11}=$ $T\left(f_{1}^{0},\left(b_{2}-\lambda_{2}\right) f_{2}^{\infty}\right)(z), c_{12}=T\left(f_{1}^{\infty},\left(b_{2}-\lambda_{2}\right) f_{2}^{0}\right)(z), c_{13}=T\left(f_{1}^{\infty},\left(b_{2}-\lambda_{2}\right) f_{2}^{\infty}\right)(z)$. Choosing $1<p<\frac{1}{2 \delta}$ and using Hölder's inequality, note that $r \leq 1$, we then obtain

$$
\begin{aligned}
I I \leq & C\left\|b_{1}\right\|_{B M O_{\theta}}\left(\frac{1}{|Q|} \int_{Q}\left|T\left(f_{1}^{0},\left(b_{2}-\lambda_{2}\right) f_{2}^{0}\right)(z)\right|^{p \delta} d z\right)^{\frac{1}{p \delta}} \\
& +C\left(\frac{1}{|Q|} \int_{Q}\left|T\left(f_{1}^{0},\left(b_{2}-\lambda_{2}\right) f_{2}^{\infty}\right)(z)-c_{11}\right|^{p \delta} d z\right)^{\frac{1}{p \delta}} \\
& +C\left(\frac{1}{|Q|} \int_{Q}\left|T\left(f_{1}^{\infty},\left(b_{2}-\lambda_{2}\right) f_{2}^{0}\right)(z)-c_{12}\right|^{p \delta} d z\right)^{\frac{1}{p \delta}} \\
& +C\left(\frac{1}{|Q|} \int_{Q}\left|T\left(f_{1}^{\infty},\left(b_{2}-\lambda_{2}\right) f_{2}^{\infty}\right)(z)-c_{13}\right|^{p \delta} d z\right)^{\frac{1}{p \delta}} \\
:= & C\left\|b_{1}\right\|_{B M O_{\theta}}\left(I I_{1}+I I_{2}+I I_{3}+I I_{4}\right) .
\end{aligned}
$$

Using $p \delta<\frac{1}{2}$, Kolmogorov inequality and (2.2)

$$
\begin{aligned}
I I_{1} & \leq C\left\|T\left(f_{1}^{0},\left(b_{2}-\lambda_{2}\right) f_{2}^{0}\right)\right\|_{L^{\frac{1}{2}, \infty}\left(Q, \frac{d x}{|Q|}\right)} \\
& \leq C \frac{1}{|Q|} \int_{Q}\left|f_{1}^{0}(z)\right| d z \frac{1}{|Q|} \int_{Q}\left|\left(b_{2}(z)-\lambda_{2}\right) f_{2}^{0}(z)\right| d z \\
& \leq C\left|f_{1}\right| Q\left\|b_{2}\right\|_{B M O_{\theta}}\left\|f_{2}\right\|_{L(\log L), Q}(1+r)^{\theta} \\
& \leq C\left\|b_{2}\right\|_{B M O_{\theta}} \mathbb{M}_{L(\log L), \varphi, \eta}\left(f_{1}, f_{2}\right)(x) .
\end{aligned}
$$

Since $I I_{2}$ and $I I_{3}$ are symmetric, we only estimate the first one. By Proposition 2.5, we have

$$
\int_{3^{k+1} Q}\left|b_{2}-\lambda_{2}\right|\left|f_{2}\left(y_{2}\right)\right| d y_{2} \leq C k\left(1+3^{k+1} r\right)^{\theta}\left\|b_{2}\right\|_{B M O_{\theta}}\left|3^{k+1} Q\right|\left\|f_{2}\right\|_{L(\log L), 3^{k+1} Q} .
$$


When taking $N=\theta+2 \eta$, we get

$$
\begin{aligned}
I I_{2} \leq & C \frac{1}{|Q|} \int_{Q}\left|T\left(f_{1}^{0},\left(b_{2}-\lambda_{2}\right) f_{2}^{\infty}\right)(z)-T\left(f_{1}^{0},\left(b_{2}-\lambda_{2}\right) f_{2}^{\infty}\right)(x)\right| d z \\
\leq & C \frac{1}{|Q|} \int_{Q}\left(\int_{3 Q}\left|f_{1}\left(y_{1}\right)\right| d y_{1}\right) \\
& \times\left(\int_{\left(\mathbb{R}^{n} \backslash 3 Q\right)} \frac{|x-z|^{\varepsilon}\left|b_{2}\left(y_{2}\right)-\lambda_{2}\right|\left|f_{2}\right| d y_{2}}{\left(\left|z-y_{1}\right|+\left|z-y_{2}\right|\right)^{2 n+\varepsilon}\left(1+\left|z-y_{1}\right|+\left|z-y_{2}\right|\right)^{N}}\right) d z \\
\leq & C\left(\int_{3 Q}\left|f_{1}\left(y_{1}\right)\right| d y_{1}\right)\left(\sum_{k=1}^{\infty} \frac{|Q|^{\frac{\varepsilon}{n}}}{\left(3^{k}|Q|^{\frac{1}{n}}\right)^{2 n+\varepsilon}\left(1+3^{k}|Q|^{\frac{1}{n}}\right)^{N}} \int_{3^{k+1} Q}\left|b_{2}-\lambda_{2}\right|\left|f_{2}\right| d y_{2}\right) \\
\leq & \left\|b_{2}\right\|_{B M O_{\theta}} \sum_{k=1}^{\infty} k 3^{-k \varepsilon} \frac{\left(1+3^{k+1} r\right)^{\theta}}{\left(1+3^{k} r\right)^{N}}\left|f_{1}\right|_{3^{k+1} Q}\left|f_{2}\right|_{L(\log L), 3^{k+1} Q} \\
\leq & \|\|_{2} \|_{B M O_{\theta}} \mathbb{M}_{L(\log L), \varphi, \eta}\left(f_{1}, f_{2}\right)(x) .
\end{aligned}
$$

For $I I_{4}$, using Proposition 2.5 and (4.2) and taking $N=2 \eta+\theta$, we have

$$
\begin{aligned}
I I_{4} & \leq C \frac{1}{|Q|}\left|T\left(f_{1}^{\infty},\left(b_{2}-\lambda_{2}\right) f_{2}^{\infty}\right)(z)-T\left(f_{1}^{\infty},\left(b_{2}-\lambda_{2}\right) f_{2}^{\infty}\right)(x)\right| d z \\
& \leq C \frac{1}{|Q|} \int_{Q}\left(\int_{\left(\mathbb{R}^{n} \backslash 3 Q\right)^{2}} \frac{\left|f_{1}\left(y_{1}\right)\left(b_{2}-\lambda_{2}\right) f_{2}\left(y_{2}\right)\right||x-z|^{\varepsilon}}{\left(\left|z-y_{1}\right|+\left|z-y_{2}\right|\right)^{2 n+\varepsilon}\left(1+\left|z-y_{1}\right|+\left|z-y_{2}\right|\right)^{N}} d y_{1} d y_{2}\right) d z \\
& \leq C \sum_{k=1}^{\infty} \frac{|Q|^{\frac{\varepsilon}{n}}}{\left(3^{k}|Q|^{\frac{1}{n}}\right)^{2 n+\varepsilon}\left(1+3^{k}|Q|^{\frac{1}{n}}\right)^{N}} \int_{3^{k+1} Q}\left|f_{1}\left(y_{1}\right)\right| d y_{1} \int_{3^{k+1} Q}\left|b_{2}\left(y_{2}\right)-\lambda_{2}\right|\left|f_{2}\left(y_{2}\right)\right| d y_{2} \\
& \leq C\left\|b_{2}\right\|_{B M O_{\theta}} \sum_{k=1}^{\infty} k 3^{-k \varepsilon} \frac{\left(1+3^{k+1} r\right)^{2 \eta+\theta}}{\left(1+3^{k} r\right)^{N}} \mathbb{M}_{L(\log L), \varphi, \eta}\left(f_{1}, f_{2}\right)(x) \\
& \leq C\left\|b_{2}\right\|_{B M O_{\theta}} \mathbb{M}_{L(\log L), \varphi, \eta}\left(f_{1}, f_{2}\right)(x) .
\end{aligned}
$$

We now estimate $I V$.

$$
\begin{aligned}
I V \leq & C\left(\frac{1}{|Q|} \int_{Q}\left|T\left(\left(b_{1}-\lambda_{1}\right) f_{1}^{0},\left(b_{2}-\lambda_{2}\right) f_{2}^{0}\right)(z)\right|^{\delta} d z\right)^{\frac{1}{\delta}} \\
& +C\left(\frac{1}{|Q|} \int_{Q}\left|T\left(\left(b_{1}-\lambda_{1}\right) f_{1}^{0},\left(b_{2}-\lambda_{2}\right) f_{2}^{\infty}\right)(z)-c_{31}\right|^{\delta} d z\right)^{\frac{1}{\delta}} \\
& +C\left(\frac{1}{|Q|} \int_{Q}\left|T\left(\left(b_{1}-\lambda_{1}\right) f_{1}^{\infty},\left(b_{2}-\lambda_{2}\right) f_{2}^{0}\right)(z)-c_{32}\right|^{\delta} d z\right)^{\frac{1}{\delta}} \\
& +C\left(\frac{1}{|Q|} \int_{Q}\left|T\left(\left(b_{1}-\lambda_{1}\right) f_{1}^{\infty},\left(b_{2}-\lambda_{2}\right) f_{2}^{\infty}\right)(z)-c_{33}\right|^{\delta} d z\right)^{\frac{1}{\delta}} \\
:= & I V_{1}+I V_{2}+I V_{3}+I V_{4},
\end{aligned}
$$

where

$$
\begin{aligned}
& c_{31}=T\left(\left(b_{1}-\lambda_{1}\right) f_{1}^{0},\left(b_{2}-\lambda_{2}\right) f_{2}^{\infty}\right)(x), \\
& c_{32}=T\left(\left(b_{1}-\lambda_{1}\right) f_{1}^{\infty},\left(b_{2}-\lambda_{2}\right) f_{2}^{0}\right)(x), \\
& c_{33}=T\left(\left(b_{1}-\lambda_{1}\right) f_{1}^{\infty},\left(b_{2}-\lambda_{2}\right) f_{2}^{\infty}\right)(x) .
\end{aligned}
$$


The estimate of $I V_{1}$ is similar to $I I_{1}$, we obtain

$$
\begin{aligned}
I V_{1} & \leq C\left\|T\left(\left(b_{1}-\lambda_{1}\right) f_{1}^{0},\left(b_{2}-\lambda_{2}\right) f_{2}^{0}\right)\right\|_{L^{\frac{1}{2}, \infty}\left(Q, \frac{d x}{|Q|}\right)} \\
& \leq C \frac{1}{|Q|} \int_{Q}\left|\left(b_{1} z-\lambda_{1}\right) f_{1}^{0}(z)\right| d z \frac{1}{|Q|} \int_{Q}\left|\left(b_{2}(z)-\lambda_{2}\right) f_{2}^{0}(z)\right| d z \\
& \leq C(1+r)^{2 \theta}\left\|b_{1}\right\|_{B M O_{\theta}}\left\|b_{2}\right\|_{B M O_{\theta}}\left\|f_{1}\right\|_{L(\log L), Q}\left\|f_{2}\right\|_{L(\log L), Q} \\
& \leq C\left\|b_{1}\right\|_{B M O_{\theta}}\left\|b_{2}\right\|_{B M O_{\theta}} \mathbb{M}_{L(\log L), \varphi, \eta}\left(f_{1}, f_{2}\right)(x) .
\end{aligned}
$$

Since $I V_{2}$ and $I V_{3}$ are symmetric, we only study the first one.

$$
\begin{aligned}
& I V_{2} \leq C \frac{1}{|Q|} \int_{Q}\left|T\left(\left(b_{1}-\lambda_{1}\right) f_{1}^{0},\left(b_{2}-\lambda_{2}\right) f_{2}^{\infty}\right)(z)-T\left(\left(b_{1}-\lambda_{1}\right) f_{1}^{0},\left(b_{2}-\lambda_{2}\right) f_{2}^{\infty}\right)(x)\right| d z \\
& \leq C \frac{1}{|Q|} \int_{Q} \int_{3 Q}\left|\left(b_{1}\left(y_{1}\right)-\lambda_{1}\right) f_{1}\left(y_{1}\right)\right| d y_{1} \\
& \times \int_{\mathbb{R}^{n} \backslash 3 Q} \frac{|x-z|^{\varepsilon}\left|\left(b_{2}\left(y_{2}\right)-\lambda_{2}\right) f_{2}\left(y_{2}\right)\right|}{\left(\left|z-y_{1}\right|+\left|z-y_{2}\right|\right)^{2 n+\varepsilon}\left(1+\left|z-y_{1}\right|+\left|z-y_{2}\right|\right)^{N}} d z \\
& \leq C \sum_{k=1}^{\infty} \frac{|Q|^{\frac{\varepsilon}{n}}}{\quad} \frac{\left(\int_{3^{k+1} Q}|Q|^{\frac{1}{n}}\right)^{2 n+\varepsilon}\left(1+3^{k}|Q|^{\frac{1}{n}}\right)^{N}}{\left.\left.\mid\left(b_{1}\right)-\lambda_{1}\right) f_{1}\left(y_{1}\right) \mid d y_{1}\right)\left(\int_{3^{k+1} Q}\left|\left(b_{2}\left(y_{2}\right)-\lambda_{2}\right) f_{2}\left(y_{2}\right)\right| d y_{2}\right)} \\
& \leq C\left\|b_{1}\right\|_{B M O_{\theta}}\left\|b_{2}\right\|_{B M O_{\theta}} \mathbb{M}_{L(\log L), \varphi, \eta}\left(f_{1}, f_{2}\right)(x) \sum_{k=1}^{\infty} k^{2} 3^{-k \varepsilon} \frac{\left(1+3^{k+1} r\right)^{2 \theta^{\prime}+2 \theta \eta}}{\left(1+3^{k} r\right)^{N}} \\
& \leq C\left\|b_{1}\right\|_{B M O_{\theta}}\left\|b_{2}\right\|_{B M O_{\theta}} \mathbb{M}_{L(\log L), \varphi, \eta}\left(f_{1}, f_{2}\right)(x),
\end{aligned}
$$

where taking $N=2(\eta+\theta)$.

Finally, in the similar way we estimate $I V_{4}$ and obtain

$$
I V_{4} \leq C\left\|b_{1}\right\|_{B M O_{\theta}}\left\|b_{2}\right\|_{B M O_{\theta}} \mathbb{M}_{L(\log L), \varphi, \eta}\left(f_{1}, f_{2}\right)(x)
$$

Case 2: $r \geq 1$. Let $0<\delta<\varepsilon<1$. Denote $Q^{*}=3 Q$ and Let $\lambda_{j}=\left(b_{j}\right)_{Q^{*}}$ be the average of $b_{j}$ on $Q^{*}, j=1,2$. For any $1<q_{1}, q_{2}, q_{3}<\infty$ with $\frac{1}{q_{1}}+\frac{1}{q_{2}}+\frac{1}{q_{3}}=1$ and $q_{3}<\frac{\varepsilon}{\delta}$.

$$
\begin{aligned}
&\left(\frac{1}{\varphi(Q)^{\eta}|Q|} \int_{Q}\left|T_{\Pi \vec{b}}(\vec{f})(y)\right|^{\delta} d y\right)^{\frac{1}{\delta}} \\
& \leq C \frac{1}{\varphi(Q)^{\eta / \delta}}\left(\frac{1}{|Q|} \int_{Q}\left|\left(b_{1}-\lambda_{1}\right)\left(b_{2}-\lambda_{2}\right) T\left(f_{1}, f_{2}\right)(z)\right|^{\delta} d z\right)^{\frac{1}{\delta}} \\
& \quad+C \frac{1}{\varphi(Q)^{\eta / \delta}}\left(\frac{1}{|Q|} \int_{Q}\left|\left(b_{1}-\lambda_{1}\right) T\left(f_{1},\left(b_{2}-\lambda_{2}\right) f_{2}\right)(z)\right|^{\delta} d z\right)^{\frac{1}{\delta}} \\
& \quad+C \frac{1}{\varphi(Q)^{\eta / \delta}}\left(\frac{1}{|Q|} \int_{Q}\left|\left(b_{2}-\lambda_{2}\right) T\left(\left(b_{1}-\lambda_{1}\right) f_{1}, f_{2}\right)(z)\right|^{\delta} d z\right)^{\frac{1}{\delta}} \\
& \quad+C \frac{1}{\varphi(Q)^{\eta / \delta}}\left(\frac{1}{|Q|} \int_{Q}\left|T\left(\left(b_{1}-\lambda_{1}\right) f_{1},\left(b_{2}-\lambda_{2}\right) f_{2}\right)(z)\right|^{\delta} d z\right)^{\frac{1}{\delta}} \\
&:= I+I I+I I I+I V .
\end{aligned}
$$


By Hölder's inequality, choosing $\eta$ so that $\eta\left(\frac{1}{\delta}-\frac{1}{\varepsilon}\right)>2 \theta$, we have

$$
\begin{aligned}
I \leq C \frac{1}{\varphi(Q)^{\eta / \delta}}\left(\frac{1}{|Q|} \int_{Q}\left|b_{1}(z)-\lambda_{1}\right|^{q_{1} \delta} d z\right)^{\frac{1}{q_{1} \delta}} \\
\quad \times\left(\frac{1}{|Q|} \int_{Q}\left|b_{2}(z)-\lambda_{2}\right|^{q_{2} \delta} d z\right)^{\frac{1}{q_{2} \delta}}\left(\frac{1}{|Q|} \int_{Q}\left|T\left(f_{1}, f_{2}\right)\right|^{q_{3} \delta}\right)^{\frac{1}{q_{3} \delta}} \\
\leq C \frac{\varphi(Q)^{\eta / \varepsilon}}{\varphi(Q)^{\eta / \delta}}(1+3 r)^{2 \theta}\left\|b_{1}\right\|_{B M O_{\theta}}\left\|b_{2}\right\|_{B M O_{\theta}}\left(\frac{1}{\varphi(Q)^{\eta}|Q|} \int_{Q}\left|T\left(f_{1}, f_{2}\right)(z)\right|^{\varepsilon} d z\right)^{\frac{1}{\varepsilon}} \\
\leq C \frac{1}{\varphi(Q)^{\eta / \delta-\eta / \varepsilon-2 \theta}}\left\|b_{1}\right\|_{B M O_{\theta}}\left\|b_{2}\right\|_{B M O_{\theta}} M_{\varepsilon, \varphi, \eta}^{\triangle}\left(T\left(f_{1}, f_{2}\right)\right)(x) \\
\leq C\left\|b_{1}\right\|_{B M O_{\theta}}\left\|b_{2}\right\|_{B M O_{\theta}} M_{\varepsilon, \varphi, \eta}^{\triangle}\left(T\left(f_{1}, f_{2}\right)\right)(x) .
\end{aligned}
$$

By Hölder's inequality and Proposition 2.5, we have

$$
\begin{aligned}
& I I \leq \frac{1}{\varphi(Q)^{\eta / \delta}}\left(\frac{1}{|Q|} \int_{Q}\left|b_{1}(z)-\lambda_{1}\right|^{p^{\prime} \delta} d z\right)^{\frac{1}{p^{\prime} \delta}}\left(\frac{1}{|Q|} \int_{Q}\left|T\left(f_{1},\left(b_{2}-\lambda_{2}\right) f_{2}\right)(z)\right|^{p \delta} d z\right)^{\frac{1}{p \delta}} \\
& \leq C\left\|b_{1}\right\|_{B M O_{\theta}} \frac{1}{\varphi(Q)^{\eta / \delta-\theta}}\left(\frac{1}{|Q|} \int_{Q}\left|T\left(f_{1}^{0},\left(b_{2}-\lambda_{2}\right) f_{2}^{0}\right)(z)\right|^{p \delta} d z\right)^{\frac{1}{p \delta}} \\
& +C\left\|b_{1}\right\|_{B M O_{\theta}} \frac{1}{\varphi(Q)^{\eta / \delta-\theta}}\left(\frac{1}{|Q|} \int_{Q}\left|T\left(f_{1}^{0},\left(b_{2}-\lambda_{2}\right) f_{2}^{\infty}\right)(z)\right|^{p \delta} d z\right)^{\frac{1}{p \delta}} \\
& +C\left\|b_{1}\right\|_{B M O_{\theta}} \frac{1}{\varphi(Q)^{\eta / \delta-\theta}}\left(\frac{1}{|Q|} \int_{Q}\left|T\left(f_{1}^{\infty},\left(b_{2}-\lambda_{2}\right) f_{2}^{0}\right)(z)\right|^{p \delta} d z\right)^{\frac{1}{p \delta}} \\
& +C\left\|b_{1}\right\|_{B M O_{\theta}} \frac{1}{\varphi(Q)^{\eta / \delta-\theta}}\left(\frac{1}{|Q|} \int_{Q}\left|T\left(f_{1}^{\infty},\left(b_{2}-\lambda_{2}\right) f_{2}^{\infty}\right)(z)\right|^{p \delta} d z\right)^{\frac{1}{p \delta}} \\
& :=I I_{1}+I I_{2}+I I_{3}+I I_{4} \text {. }
\end{aligned}
$$

Choosing $\eta$ so that $\eta\left(\frac{1}{\delta}-2\right)-2 \theta>0$, we now estimate $I I_{1}$.

$$
\begin{aligned}
I I_{1} & \leq C\left\|b_{1}\right\|_{B M O_{\theta}} \frac{1}{\varphi(Q)^{\eta / \delta-\theta}}\left\|T\left(f_{1}^{0},\left(b_{2}-\lambda_{2}\right) f_{2}^{0}\right)\right\|_{L^{\frac{1}{2}, \infty}\left(Q, \frac{d x}{|Q|}\right)} \\
& \leq C\left\|b_{1}\right\|_{B M O_{\theta}} \frac{1}{\varphi(Q)^{\eta / \delta-\theta}} \frac{1}{|Q|} \int_{Q}\left|f_{1}^{0}(z)\right| d z \frac{1}{|Q|} \int_{Q}\left|\left(b_{2}(z)-\lambda_{2}\right) f_{2}^{0}(z)\right| d z \\
& \leq C\left\|b_{1}\right\|_{B M O_{\theta}} \frac{1}{\varphi(Q)^{\eta / \delta-\theta}}\left\|b_{2}\right\|_{B M O_{\theta}} \varphi(Q)\left|f_{1}\right| Q\left\|f_{2}\right\|_{L(\log L), Q} \\
& \leq C\left\|b_{1}\right\|_{B M O_{\theta}}\left\|b_{2}\right\|_{B M O_{\theta}} \frac{1}{\varphi(Q)^{\eta\left(\frac{1}{\delta}-2\right)-2 \theta}} \mathbb{M}_{L(\log L), \varphi, \eta}\left(f_{1}, f_{2}\right)(x) \\
& \leq C\left\|b_{1}\right\|_{B M O_{\theta}}\left\|b_{2}\right\|_{B M O_{\theta}} \mathbb{M}_{L(\log L), \varphi, \eta}\left(f_{1}, f_{2}\right)(x) .
\end{aligned}
$$


Since $I I_{2}$ and $I I_{3}$ are symmetric, we only estimate $I I_{2}$. By Proposition 2.5, (4.2) and Hölder's inequality, we have

$$
\begin{aligned}
I I_{2} \leq & C\left\|b_{1}\right\|_{B M O_{\theta}} \frac{1}{\varphi(Q)^{\eta / \delta-\theta}} \frac{1}{|Q|} \int_{Q}\left|T\left(f_{1}^{0},\left(b_{2}-\lambda_{2}\right) f_{2}^{\infty}\right)(z)\right| d z \\
\leq & C\left\|b_{1}\right\|_{B M O_{\theta}} \frac{1}{\varphi(Q)^{\eta / \delta-\theta}} \frac{1}{|Q|} \int_{Q}\left(\int_{3 Q}\left|f_{1}\left(y_{1}\right)\right| d y_{1}\right) \\
& \times\left(\int_{\mathbb{R}^{n} \backslash 3 Q} \frac{\left|\left(b_{2}\left(y_{2}\right)-\lambda_{2}\right) f_{2}\left(y_{2}\right) d y_{2}\right|}{\left(\left|z-y_{1}\right|+\left|z-y_{2}\right|\right)^{2 n}\left(1+\left|z-y_{1}\right|+\left|z-y_{2}\right|\right)^{N}}\right) d z \\
\leq & C\left\|b_{1}\right\|_{B M O_{\theta}} \frac{1}{\varphi(Q)^{\eta / \delta-\theta}} \sum_{k=1}^{\infty} \frac{1}{\left(3^{k}|Q|^{\frac{1}{n}}\right)^{2 n}} \frac{1}{\left(1+3^{k}|Q|^{\frac{1}{n}}\right)^{N}}\left(\int_{3^{k+1} Q}\left|f_{1}\left(y_{1}\right)\right| d y_{1}\right) \\
& \times\left(\int_{3^{k+1} Q}\left|\left(b_{2}\left(y_{2}\right)-\lambda 2\right)\right|\left|f_{2}\left(y_{2}\right)\right| d y_{2}\right) \\
\leq & \left\|b_{1}\right\|_{B M O_{\theta}} \frac{1}{\varphi(Q)^{\eta / \delta-\theta}}\left\|b_{2}\right\|_{B M O_{\theta}} \mathbb{M}_{L(\log L), \varphi, \eta}\left(f_{1}, f_{2}\right)(x) \sum_{k=1}^{\infty} \frac{\left(1+3^{k+1} r\right)^{\theta+2 \eta} k}{\left(1+3^{k}|Q|^{\frac{1}{n}}\right)^{N}}
\end{aligned}
$$

We need to take $N=\theta+2 \eta+1$ and choose $\eta$ so that $\eta / \delta-\theta>0$, then we obtain

$$
I I_{2} \leq C\left\|b_{1}\right\|_{B M O_{\theta}}\left\|b_{2}\right\|_{B M O_{\theta}} \mathbb{M}_{L(\log L), \varphi, \eta}\left(f_{1}, f_{2}\right)(x) .
$$

For the last term $I I_{4}$, taking $N=\theta+2 \eta+1$ and $\eta$ which satisfies $\eta / \delta-\theta>0$, we have

$$
\begin{aligned}
I_{4} \leq C & \left\|b_{1}\right\|_{B M O_{\theta}} \frac{1}{\varphi(Q)^{\eta / \delta-\theta}} \frac{1}{|Q|} \\
& \times\left(\sum_{k=1}^{\infty} \int_{\left(3^{k+1} Q\right)^{2} \backslash\left(3^{k} Q\right)^{2}} \frac{\left|f_{1}\left(y_{1}\right)\right|\left|f_{2}\left(y_{2}\right)\right|\left|b_{2}\left(y_{2}\right)-\lambda_{2}\right|}{\left(\left|z-y_{1}\right|+\left|z-y_{2}\right|\right)^{2 n}\left(1+\left|z-y_{1}\right|+\left|z-y_{2}\right|\right)^{N}}\right) d z
\end{aligned}
$$

$\leq C\left\|b_{1}\right\|_{B M O_{\theta}} \frac{1}{\varphi(Q)^{\eta / \delta-\theta}}$

$$
\times \sum_{k=1}^{\infty} \frac{1}{\left(1+3^{k}|Q|^{\frac{1}{n}}\right)^{N}\left(3^{k}|Q|^{\frac{1}{n}}\right)^{2 n}} \int_{\left(3^{k+1} Q\right)^{2}}\left|f_{1}\left(y_{1}\right)\right|\left|f_{2}\left(y_{2}\right)\right|\left|b_{2}\left(y_{2}\right)-\lambda_{2}\right| d y_{1} d y_{2}
$$

$\leq C\left\|b_{1}\right\|_{B M O_{\theta}} \frac{1}{\varphi(Q)^{\eta / \delta-\theta}}\left\|b_{2}\right\|_{B M O_{\theta}} \mathbb{M}_{L(\log L), \varphi, \eta}\left(f_{1}, f_{2}\right)(x) \sum_{k=1}^{\infty} \frac{k\left(1+3^{k+1} r\right)^{\theta+2 \eta}}{\left(1+3^{k} r\right)^{N}}$

$\leq C\left\|b_{1}\right\|_{B M O_{\theta}}\left\|b_{2}\right\|_{B M O_{\theta}} \mathbb{M}_{L(\log L), \varphi, \eta}\left(f_{1}, f_{2}\right)(x)$.

We now estimate $I V$. As before, we first split any function

$$
\begin{aligned}
I V \leq & C \frac{1}{\varphi(Q)^{\eta / \delta}}\left(\frac{1}{|Q|} \int_{Q}\left|T\left(\left(b_{1}-\lambda_{1}\right) f_{1}^{0},\left(b_{2}-\lambda_{2}\right) f_{2}^{0}\right)(z)\right|^{\delta} d z\right)^{\frac{1}{\delta}} \\
& +\frac{1}{\varphi(Q)^{\eta / \delta}}\left(\frac{1}{|Q|} \int_{Q}\left|T\left(\left(b_{1}-\lambda_{1}\right) f_{1}^{0},\left(b_{2}-\lambda_{2}\right) f_{2}^{\infty}\right)(z)\right|^{\delta} d z\right)^{\frac{1}{\delta}} \\
& +\frac{1}{\varphi(Q)^{\eta / \delta}}\left(\frac{1}{|Q|} \int_{Q}\left|T\left(\left(b_{1}-\lambda_{1}\right) f_{1}^{\infty},\left(b_{2}-\lambda_{2}\right) f_{2}^{0}\right)(z)\right|^{\delta} d z\right)^{\frac{1}{\delta}} \\
& +\frac{1}{\varphi(Q)^{\eta / \delta}}\left(\frac{1}{|Q|} \int_{Q}\left|T\left(\left(b_{1}-\lambda_{1}\right) f_{1}^{\infty},\left(b_{2}-\lambda_{2}\right) f_{2}^{\infty}\right)(z)\right|^{\delta} d z\right)^{\frac{1}{\delta}} \\
:= & I V_{1}+I V_{2}+I V_{3}+I V_{4} .
\end{aligned}
$$


The estimate of $I V_{1}$ is similar to $I I_{1}$. Taking $\eta$ so that $\eta\left(\frac{1}{\delta}-2\right)>2 \theta$, we obtain

$$
I V_{1} \leq C\left\|b_{1}\right\|_{B M O_{\theta}}\left\|b_{2}\right\|_{B M O_{\theta}} \mathbb{M}_{L(\log L), \varphi, \eta}\left(f_{1}, f_{2}\right)(x)
$$

Since $I V_{2}$ and $I V_{3}$ are symmetric, we only estimate $I V_{2}$, (similar to $\left.I I_{2}\right)$. Taking $N=$ $2 \theta+2 \theta \eta+1$, we get

$$
\begin{aligned}
I V_{2} \leq & C \frac{1}{\varphi(Q)^{\eta / \delta}} \frac{1}{|Q|} \int_{Q}\left|T\left(\left(b_{1}-\lambda_{1}\right) f_{1}^{0},\left(b_{2}-\lambda_{2}\right) f_{2}^{\infty}\right)(z)\right| d z \\
\leq & C \frac{1}{\varphi(Q)^{\eta / \delta}} \sum_{k=1}^{\infty} \frac{1}{\left(1+3^{k}|Q|^{\frac{1}{n}}\right)^{N}\left(3^{k}|Q|^{\frac{1}{n}}\right)^{2 n}}\left(\int_{3^{k+1} Q}\left|\left(b_{1}\left(y_{1}\right)-\lambda_{1}\right) f_{1}\left(y_{1}\right)\right| d y_{1}\right) \\
& \times\left(\int_{3^{k+1} Q}\left|\left(b_{2}\left(y_{2}\right)-\lambda_{2}\right) f_{2}\left(y_{2}\right)\right| d y_{2}\right) \\
\leq & C\left\|b_{1}\right\|_{B M O_{\theta}}\left\|b_{2}\right\|_{B M O_{\theta}} \mathbb{M}_{L(\log L), \varphi, \eta}\left(f_{1}, f_{2}\right)(x) \sum_{k=1}^{\infty} \frac{k^{2}\left(1+3^{k+1} r\right)^{2 \theta+2 \eta}}{\left(1+3^{k} r\right)^{N}} \\
\leq & C\left\|b_{1}\right\|_{B M O_{\theta}}\left\|b_{2}\right\|_{B M O_{\theta}} \mathbb{M}_{L(\log L), \varphi, \eta}\left(f_{1}, f_{2}\right)(x) .
\end{aligned}
$$

Finally, we estimate $I V_{4}$ in the similar way and we obtain

$$
I V_{4} \leq C\left\|b_{1}\right\|_{B M O_{\theta}}\left\|b_{2}\right\|_{B M O_{\theta}} \mathbb{M}_{L(\log L), \varphi, \eta}\left(f_{1}, f_{2}\right)(x)
$$

From the estimates above, we see that it is enough to take $\eta \geq 2 \theta /(1 / \delta-1 / \varepsilon)$. The proof is complete.

We note that we can also obtain analogous estimates to (4.1) for $m$-linear commutators involving, $j<m$ function $B M O_{\theta}$.

Proposition 4.2 Let $p>0$ and $\omega$ be a weight in $A_{\infty}^{\infty}$. Suppose that $T_{\Pi \vec{b}}$ is a multilinear commutator with $\vec{b} \in B M O_{\vec{\theta}}^{m}\left(\vec{\theta}=\left(\theta_{1}, \cdots, \theta_{m}\right)\right)$ and $T$ satisfying (1.1)-(1.4). Let $\eta>0$. Then there exists a constant $C>0$ depending on the $A_{\infty}^{\infty}$ constant of $\omega$ such that

$$
\int_{\mathbb{R}^{n}}\left|T_{\Pi \vec{b}}(\vec{f})(x)\right|^{p} \omega(x) d x \leq C \prod_{i=1}^{m}\left\|b_{i}\right\|_{B M O_{\theta_{i}}} \int_{\mathbb{R}^{n}} \mathbb{M}_{L(\log L), \varphi, \eta}(\vec{f})(x)^{p} \omega(x) d x
$$

and

$$
\begin{aligned}
& \sup _{t>0} \frac{1}{\Phi^{(m)}\left(\frac{1}{t}\right)} \omega\left(\left\{y \in \mathbb{R}^{n}:\left|T_{\Pi \vec{b}}(\vec{f})(y)\right|>t^{m}\right\}\right) \\
& \leq C \sup _{t>0} \frac{1}{\Phi^{(m)}\left(\frac{1}{t}\right)} \omega\left(\left\{y \in \mathbb{R}^{n}: \mathbb{M}_{L(\log L), \varphi, \eta}(\vec{f})(y)>t^{m}\right\}\right)
\end{aligned}
$$

for all $\vec{f}=\left(f_{1}, \cdots, f_{m}\right)$ bounded with compact support.

Proof Applying Proposition 2.8, Theorem 3.1 and Proposition 4.1, in the similar way as in Theorem 3.2 in [11], we can get the estimates above. We omit the details here. 
Theorem 4.1 Let $T_{\Pi \vec{b}}$ be a multilinear commutator with $\vec{b} \in B M O_{\vec{\theta}}^{m}(\vec{\theta}=$ $\left.\left(\theta_{1}, \cdots, \theta_{m}\right)\right)$ and $T$ satisfying (1.1)-(1.4). Let $\vec{w} \in A_{\vec{p}}^{\infty}$ with $\frac{1}{p}=\frac{1}{p_{1}}+\cdots+\frac{1}{p_{m}}$ and $1<p_{j}<\infty, j=1, \cdots, m$. Then, there exists a constant $C$ such that

$$
\left\|T_{\Pi \vec{b}}(\vec{f})\right\|_{L^{p}\left(v_{\vec{\omega}}\right)} \leq C \prod_{j=1}^{m}\left\|b_{j}\right\|_{B M O_{\theta_{j}}} \prod_{j=1}^{m}\left\|f_{j}\right\|_{L^{p_{j}\left(\omega_{j}\right)}} .
$$

Proof Since $\vec{\omega} \in A_{\vec{p}}^{\infty}$, by Proposition 2.3 we get the weight $\nu_{\vec{\omega}}$ is in $A_{p m}^{\infty}$. Applying Proposition 4.2, we have that

$$
\int_{\mathbb{R}^{n}}\left|T_{\Pi \vec{b}}(\vec{f})(x)\right|^{p} \nu_{\vec{\omega}}(x) d x \leq C \prod_{j=1}^{m}\left\|b_{j}\right\|_{B M O_{\theta_{j}}} \int_{\mathbb{R}^{n}} \mathbb{M}_{L(\log L), \varphi, \eta}(\vec{f})(x)^{p} v_{\vec{\omega}}(x) d x .
$$

If $\mu>1$ and since $\Phi(t)=t\left(1+\log ^{+}(t)\right) \leq t^{\mu}, t>1$, we have

$$
\|f\|_{L(\log L), Q \leq C}\left(\frac{1}{|Q|} \int_{Q}|f(y)|^{\mu} d y\right)^{\frac{1}{\mu}} .
$$

Thus, we have

$$
\begin{aligned}
\frac{1}{\varphi(Q)^{\eta}}\|f\|_{L(\log L), Q} & \leq C \frac{1}{\varphi(Q)^{\eta}}\left(\frac{1}{|Q|} \int_{Q}|f(y)|^{\mu} d y\right)^{\frac{1}{\mu}} \\
& \leq C\left(\frac{1}{\varphi(Q)^{\eta}|Q|} \int_{Q}|f(y)|^{\mu} d y\right)^{\frac{1}{\mu}},
\end{aligned}
$$

and $\mathbb{M}_{L(\log L), \varphi, \eta} \vec{f}(x) \leq \mathbb{M}_{\mu, \varphi, \eta}(\vec{f})(x)$. Now we obtain

$$
\left\|T_{\Pi \vec{b}}(\vec{f})\right\|_{L^{p}\left(v_{\vec{\omega}}\right)} \leq C\left\|\mathbb{M}_{\mu, \varphi, \eta}(\vec{f})\right\|_{L^{p}\left(v_{\vec{\omega}}\right)},
$$

Now to prove $\left\|\mathbb{M}_{\mu, \varphi, \eta}(\vec{f})\right\|_{L^{p}\left(\nu_{\vec{\omega}}\right)} \leq C \prod_{j=1}^{m}\left\|f_{j}\right\|_{L^{p_{j}\left(\omega_{j}\right)}}$ is equivalent to prove $\|$ $\mathbb{M}_{\varphi, \eta}(\vec{f})\left\|_{L^{p / \mu}\left(\nu_{\vec{\omega}}\right)} \leq C \prod_{j=1}^{m}\right\| f_{j} \|_{L^{p_{j}\left(\omega_{j}\right)}}$. Taking $\eta=\eta_{0}$, by Theorem 3.3, this is equivalent to prove that $\vec{\omega} \in A_{\vec{p} / \mu}^{\infty}$. In fact, we already know that this is ture for some $\mu>1$ because of Proposition 2.4(ii). Thus we get

$$
\left\|T_{\Pi \vec{b}}(\vec{f})\right\|_{L^{p}\left(v_{\vec{\omega}}\right)} \leq C \prod_{j=1}^{m}\left\|b_{j}\right\|_{B M O_{\theta_{j}}} \prod_{j=1}^{m}\left\|f_{j}\right\|_{L^{p_{j}}}\left(\omega_{j}\right) .
$$

Thus, Theorem 4.1 is proved.

Proposition 4.3 Let $\vec{\omega} \in A_{\overrightarrow{1}}^{\theta}$ and $\eta>2 \theta$. Then there exists a constant $C$ such that

$$
\nu_{\omega}\left\{x \in \mathbb{R}^{n}: \mathbb{M}_{L(\log L), \varphi, \eta}(\vec{f})(x)>t^{m}\right\} \leq C \prod_{j=1}^{m}\left(\int_{\mathbb{R}^{n}} \Phi^{(m)}\left(\frac{\left|f_{j}(x)\right|}{t}\right) \omega_{j}(x) d x\right)^{\frac{1}{m}},
$$

where $\Phi(t)=t\left(1+\log ^{+} t\right)$ and $\Phi^{(m)}=\underbrace{\Phi \circ \cdots \circ \Phi}_{m}$. 
Proof Our goal is to estimate $|\Omega|=\left|\left\{\mathbb{M}_{L(\log L), \varphi, \eta}(\vec{f})(x)>1\right\}\right|$. The set $\Omega$ is open and we may assume it to be not empty. It is enough to control the size of every compact set $F$ contained in $\Omega$. Denote $\|f\|_{\Phi, \varphi, \eta, Q}=\frac{1}{\varphi(Q)^{\eta}}\|f\|_{\Phi, Q}$.

For $x \in F$, there exists a cube $Q$ with $x \in Q$ such that

$$
\prod_{j=1}^{m}\left\|f_{j}\right\|_{\Phi, \varphi, \eta, Q}>1
$$

Thus, by Vitaly covering lemma, we can extract a finite family of disjoint cubes $\left\{Q_{i}\right\}$ such that $F \subseteq \cup_{i} 3 Q_{i}$ and $\left\{Q_{i}\right\}$ satisfies $\prod_{j=1}^{m}\left\|f_{j}\right\|_{\Phi, \varphi, \eta, Q_{i}}>1$. Let $C_{h}^{m}$ denote the family of all subset $\sigma=(\sigma(1), \cdots, \sigma(h))$ from the index $\{1, \cdots, m\}$ with $1 \leq h \leq m$ different elements. Given $\sigma \in C_{h}^{m}$ and a cube $Q_{i}$, we say that $i \in B_{\sigma}$ if $\left\|f_{\sigma(k)}\right\|_{\Phi, \varphi, \eta, Q_{i}}>1$ for $k=1, \cdots, h$ and $\left\|f_{\sigma(k)}\right\|_{\Phi, \varphi, \eta, Q_{i}} \leq 1$ for $k=h+1, \cdots, m$.

Now we consider $\sigma \in C_{h}^{m}$ and $i \in B_{\sigma}$. Denote $\prod_{k}=\prod_{j=1}^{k}\left\|f_{\sigma(j)}\right\|_{\Phi, \varphi, \eta, Q_{i}}$ and $\prod_{0}=1$. Then it is easy to check that $\prod_{k}>1$ for every $1 \leq k \leq m$. It follows that

$$
1<\Pi_{k}=\left\|f_{\sigma(k)}\right\|_{\Phi, \varphi, \eta, Q_{i}} \Pi_{k-1}=\frac{1}{\varphi\left(Q_{i}\right)^{\eta}}\left\|f_{\sigma(k)} \Pi_{k-1}\right\|_{\Phi, Q_{i}} .
$$

Since $\Phi(t)=t\left(1+\log ^{+} t\right)$, from $1<\frac{1}{\varphi\left(Q_{i}\right)^{\eta}}\left\|f_{\sigma(k)} \Pi_{k-1}\right\|_{\Phi, Q_{i}}$ we get that

$$
1<\frac{1}{\varphi\left(Q_{i}\right)^{\eta}\left|Q_{i}\right|} \int_{Q_{i}} \Phi\left(f_{\sigma(k)} \Pi_{k-1}\right)
$$

In particular, taking $k=m$, we have

$$
1<\frac{1}{\varphi\left(Q_{i}\right)^{\eta}\left|Q_{i}\right|} \int_{Q_{i}} \Phi\left(f_{\sigma(m)} \Pi_{m-1}\right) \leq \frac{1}{\varphi\left(Q_{i}\right)^{\eta}\left|Q_{i}\right|} \int_{Q_{i}} \Phi\left(f_{\sigma(m)}\right) \Phi\left(\Pi_{m-1}\right) .
$$

From the following equivalence

$$
\|f\|_{\Phi, Q} \simeq \inf _{\mu>0}\left\{\mu+\frac{\mu}{|Q|} \int_{Q} \Phi(|f| / \mu)\right\},
$$

We have

$$
\|f\|_{\Phi, \varphi, \eta, Q}=\frac{1}{\varphi(Q)^{\eta}}\|f\|_{\Phi, Q} \simeq \inf _{\mu>0}\left\{\frac{\mu}{\varphi(Q)^{\eta}}+\frac{\mu}{\varphi(Q)^{\eta}|Q|} \int_{Q} \Phi(|f| / \mu)\right\} .
$$

If $1 \leq j \leq m-h-1$, by (4.5) we get

$$
\begin{aligned}
\Phi^{j}\left(\Pi_{m-j}\right) & =\Phi^{j}\left(\left\|f_{\sigma(m-j)} \Pi_{m-j-1}\right\| \Phi, Q_{i}\right) \\
& \leq C \Phi^{j}\left(\frac{1}{\varphi\left(Q_{i}\right)^{\eta}} \cdot 1+\frac{1}{\varphi\left(Q_{i}\right)^{\eta}\left|Q_{i}\right|} \int_{Q_{i}} \Phi\left(f_{\sigma(m-j)} \Pi_{m-j-1}\right)\right) \\
& \leq C \Phi^{j}\left(\frac{1}{\varphi\left(Q_{i}\right)^{\eta}\left|Q_{i}\right|} \int_{Q_{i}} \Phi\left(f_{\sigma(m-j)} \Pi_{m-j-1}\right)\right) .
\end{aligned}
$$


From (4.6), by iterating the inequality above, since $\Phi$ is submultiplicative, we obtain

$$
\begin{aligned}
1 & <C \frac{1}{\varphi\left(Q_{i}\right)^{\eta}\left|Q_{i}\right|} \int_{Q_{i}} \Phi\left(f_{\sigma(m)}\right) \Phi\left(\Pi_{m-1}\right) \\
& \leq C \frac{1}{\varphi\left(Q_{i}\right)^{\eta}\left|Q_{i}\right|} \int_{Q_{i}}^{i} \Phi\left(f_{\sigma(m)}\right) \frac{1}{\varphi\left(Q_{i}\right)^{\eta}\left|Q_{i}\right|} \int_{Q_{i}} \Phi^{2}\left(f_{\sigma(m-1)}\right) \Phi^{2}\left(\Pi_{m-2}\right) \\
& \leq C\left(\prod_{j=0}^{m-h-1} \frac{1}{\varphi\left(Q_{i}\right)^{\eta}\left|Q_{i}\right|} \int_{Q_{i}} \Phi^{j+1}\left(f_{\sigma(m-j)}\right)\right) \Phi^{m-h}\left(\Pi_{h}\right) \\
& \leq C\left(\prod_{j=0}^{m-h-1} \frac{1}{\varphi\left(Q_{i}\right)^{\eta}\left|Q_{i}\right|} \int_{Q_{i}} \Phi^{j+1}\left(f_{\sigma(m-j)}\right)\right)\left(\prod_{j=1}^{h} \Phi^{m-h}\left(\left\|f_{\sigma(j)}\right\|_{\Phi, \varphi, \eta, Q_{i}}\right)\right) .
\end{aligned}
$$

Since $i \in B_{\sigma}$, we have $\left\|f_{\sigma(j)}\right\|_{\Phi, \varphi, \eta, Q_{i}}>1$, for $j=1, \cdots, h$, and it follows

$$
1<C\left(\prod_{j=0}^{m-h-1} \frac{1}{\varphi\left(Q_{i}\right)^{\eta}\left|Q_{i}\right|} \int_{Q_{i}} \Phi^{j+1}\left(f_{\sigma(m-j)}\right)\right)\left(\prod_{j=1}^{h} \frac{1}{\varphi\left(Q_{i}\right)^{\eta}\left|Q_{i}\right|} \int_{Q_{i}} \Phi^{m-h-1}\left(f_{\sigma(j)}\right)\right)
$$

Since for $1 \leq h \leq m$ and $0 \leq j \leq m-h-1$, we obtain that $\Phi^{j+1}(t) \leq \Phi^{m-h}(t) \leq \Phi^{m}(t)$ and $\Phi^{m-h+1}(t) \leq \Phi^{m}(t)$. From (4.7) we obtain that

$$
1<C \prod_{j=1}^{m} \frac{1}{\varphi\left(Q_{i}\right)^{\eta}\left|Q_{i}\right|} \int_{Q_{i}} \Phi^{m}\left(f_{j}\right)
$$

or

$$
\varphi\left(Q_{i}\right)^{\eta}\left|Q_{i}\right| \leq C \prod_{j=1}^{m}\left(\int_{Q_{i}} \Phi^{m}\left(f_{j}\right)\right)^{\frac{1}{m}}
$$

Since $\eta \geq 2 \theta$, we obtain that

$$
\varphi\left(Q_{i}\right)^{2 \theta}\left|Q_{i}\right| \leq C \prod_{j=1}^{m}\left(\int_{Q_{i}} \Phi^{m}\left(f_{j}\right)\right)^{\frac{1}{m}}
$$

Applying Proposition 2.2(iii) and (4.8) we obtain that

$$
\begin{aligned}
\nu_{\vec{\omega}}\left(3 Q_{i}\right) & \leq C \varphi\left(Q_{i}\right)^{\theta} \nu_{\vec{\omega}}\left(Q_{i}\right) \\
& =C \varphi\left(Q_{i}\right)^{2 \theta}\left|Q_{i}\right| \frac{\nu_{\vec{\omega}}\left(Q_{i}\right)}{\varphi\left(Q_{i}\right)^{\theta}\left|Q_{i}\right|} \\
& \leq C\left(\prod_{j=1}^{m} \int_{Q_{i}} \Phi^{m}\left(f_{j}\right)\right)^{\frac{1}{m}} \prod_{j=1}^{m}\left(\inf _{x \in Q_{i}} \omega_{j}(x)\right)^{\frac{1}{m}} \\
& \leq C \prod_{j=1}^{m}\left(\int_{Q_{i}} \Phi^{m}\left(f_{j}\right) \omega_{j}\right)^{\frac{1}{m}} .
\end{aligned}
$$


By (4.9) and Hölder's inequality at discrete level, we have

$$
\begin{aligned}
\nu_{\vec{\omega}}(F)^{m} & \leq C\left(\sum_{i} \nu_{\vec{\omega}}\left(3 Q_{i}\right)\right)^{m} \\
& \leq C\left(\sum_{h=1}^{m} \sum_{\sigma \in C_{h}^{m}} \sum_{i \in B_{\sigma}} \prod_{j=1}^{m}\left(\int_{Q_{i}} \Phi^{m}\left(f_{j} \omega_{j}(y)\right) d y\right)^{\frac{1}{m}}\right)^{m} \\
& \leq C \prod_{j=1}^{m} \int_{\mathbb{R}^{n}} \Phi^{m}\left(f_{j}(y) \omega_{j}(y)\right) d y,
\end{aligned}
$$

which concludes the proof of (4.3). The proof is complete.

In the end-point case, we obtain the following result.

Theorem 4.2 Let $T_{\Pi \vec{b}}$ be a multilinear commutator with $\vec{b} \in B M O_{\vec{\theta}}^{m}(\vec{\theta}=$ $\left.\left(\theta_{1}, \cdots, \theta_{m}\right)\right)$ and $T$ satisfying (1.1)-(1.4). Let $\vec{\omega} \in A_{\overrightarrow{1}}^{\infty}$. Then there exists a constant $C$ such that

$$
\nu_{\vec{\omega}}\left(\left\{x \in \mathbb{R}^{n}:\left|T_{\Pi \vec{b}}(\vec{f})(x)\right|>t^{m}\right\}\right) \leq C \prod_{j=1}^{m}\left(\int_{\mathbb{R}^{n}} \Phi^{(m)}\left(\frac{\left|f_{j}(x)\right|}{t}\right) \omega_{j}(x) d x\right)^{\frac{1}{m}},
$$

where $\Phi(t)=t\left(1+\log ^{+} t\right)$ and $\Phi^{(m)}=\underbrace{\Phi \circ \cdots \circ \Phi}_{m}$.

Proof By homogeneity it is enough to assume $t=1$ and hence we must prove

$$
\nu_{\vec{\omega}}\left(\left\{x \in \mathbb{R}^{n}:\left|T_{\Pi \vec{b}}(\vec{f})(x)\right|>1\right\}\right)^{m} \leq C \prod_{j=1}^{m}\left(\int_{\mathbb{R}^{n}} \Phi^{(m)}\left(\left|f_{j}(x)\right|\right) \omega_{j}(x) d x\right) .
$$

Now, since $\Phi$ is submultiplicative, we have by Propositions 4.2 and 4.3,

$$
\begin{aligned}
\nu_{\vec{\omega}} & \left(\left\{x \in \mathbb{R}^{n}:\left|T_{\Pi \vec{b}}(\vec{f})(x)\right|>1\right\}\right)^{m} \\
& \leq C \sup _{t>0} \frac{1}{\Phi^{(m)}\left(\frac{1}{t}\right)^{m}} \nu_{\vec{\omega}}\left(\left\{x \in \mathbb{R}^{n}:\left|T_{\Pi \vec{b}}(\vec{f})(x)\right|>t^{m}\right\}\right)^{m} \\
& \leq C \sup _{t>0} \frac{1}{\Phi^{(m)}\left(\frac{1}{t}\right)^{m}} \nu_{\vec{\omega}}\left(\left\{x \in \mathbb{R}^{n}: \mathbb{M}_{L(\log L), \varphi, \eta}(\vec{f})(x)>t^{m}\right\}\right)^{m} \\
& \leq C \sup _{t>0} \frac{1}{\Phi^{(m)}\left(\frac{1}{t}\right)^{m}} \prod_{j=1}^{m} \int_{\mathbb{R}^{n}} \Phi^{(m)}\left(\frac{\left|f_{j}(x)\right|}{t}\right) \omega_{j}(x) d x \\
& \leq C \sup _{t>0} \frac{1}{\Phi^{(m)}\left(\frac{1}{t}\right)^{m}} \prod_{j=1}^{m} \int_{\mathbb{R}^{n}} \Phi^{(m)}\left(\left|f_{j}(x)\right|\right) \Phi^{m}\left(\frac{1}{t}\right) \omega_{j}(x) d x \\
& \leq C \prod_{j=1}^{m} \int_{\mathbb{R}^{n}} \Phi^{(m)}\left(\left|f_{j}(x)\right|\right) \omega_{j}(x) d x .
\end{aligned}
$$

The proof is complete.

Finally, we consider the commutator $T_{\Sigma \vec{b}}$. Similar to the proof of Proposition 4.1 and the linearity of $T_{\Sigma \vec{b}}$, we get 
Corollary 4.1 Let $T_{\sum} \vec{b}$ be a multilinear commutator with $\vec{b} \in B M O_{\vec{\theta}}^{m}(\vec{\theta}=$ $\left.\left(\theta_{1}, \cdots, \theta_{m}\right)\right)$. Let $0<\delta<\epsilon<1 / m$ and $\geq 2\left(\theta_{1}+\cdots+\theta_{m}\right) /(1 \delta-1 / \epsilon)$. Then there exists a constant $C>0$, depending on $\delta$ and $\varepsilon$ such that

$$
M_{\delta, \varphi, \eta}^{\sharp, \Delta}\left(T_{\Sigma} \vec{b}(\vec{f})\right)(x) \leq C \sum_{j=1}^{m}\left\|b_{j}\right\|_{B M O_{\theta_{j}}}\left(\sum_{i=1}^{m} \mathbb{M}_{L(\log L), \varphi, \eta}^{i}(\vec{f})(x)+M_{\varepsilon, \varphi, \eta}^{\triangle}(T(\vec{f}))(x)\right)
$$

for all m-tuples $\vec{f}=\left(f_{1}, \cdots, f_{m}\right)$ of bounded measurable functions with compact support.

By Corollary 4.1 and the linearity of $T_{\Sigma \vec{b}}$, we have

Corollary 4.2 Let $T_{\Sigma \vec{b}}$ be a multilinear commutator with $\vec{b} \in B M O_{\vec{\theta}}^{m}(\vec{\theta}=$ $\left.\left(\theta_{1}, \cdots, \theta_{m}\right)\right)$ and $T$ satisfying (1.1)-(1.4). Let $\vec{w} \in A_{\vec{p}}^{\infty}$ with $\frac{1}{p}=\frac{1}{p_{1}}+\cdots+\frac{1}{p_{m}}$ and $1<p_{j}<\infty, j=1, \cdots, m$. Then, there exists a constant $C$ such that

$$
\left\|T_{\Sigma \vec{b}}(\vec{f})\right\|_{L^{p}\left(v_{\vec{\omega}}\right)} \leq C \sum_{j=1}^{m}\left\|b_{j}\right\|_{B M O_{\theta_{j}}} \prod_{j=1}^{m}\left\|f_{j}\right\|_{L^{p_{j}\left(\omega_{j}\right)}} .
$$

We remark that Corollary 4.2 has been proved in [2].

Corollary 4.3 Let $T_{\Sigma \vec{b}}$ be a multilinear commutator with $\vec{b} \in B M O_{\vec{\theta}}^{m}(\vec{\theta}=$ $\left.\left(\theta_{1}, \cdots, \theta_{m}\right)\right)$ and $T$ satisfying (1.1)-(1.4). Let $p>0$ and $\omega$ be a weight in $A_{\infty}^{\infty}$. Then there exists a constant $C>0$ depending on the $A_{\infty}^{\infty}$ constant of $\omega$ such that

$$
\int_{\mathbb{R}^{n}}\left|T_{\Sigma \vec{b}}(\vec{f})(x)\right|^{p} \omega(x) d x \leq C \sum_{i=1}^{m}\left\|b_{i}\right\|_{B M O_{\theta_{j}}} \int_{\mathbb{R}^{n}} \sum_{i=1}^{m} \mathbb{M}_{L(\log L), \varphi, \eta}^{i}(\vec{f})(x)^{p} \omega(x) d x
$$

and

$$
\begin{aligned}
\sup _{t>0} \frac{1}{\Phi\left(\frac{1}{t}\right)} \omega(\{y & \left.\left.\in \mathbb{R}^{n}:\left|T_{\Sigma \vec{b}}(\vec{f})(y)\right|>t^{m}\right\}\right) \\
& \leq C \sup _{t>0} \frac{1}{\Phi\left(\frac{1}{t}\right)} \omega\left(\left\{y \in \mathbb{R}^{n}: \sum_{i=1}^{m} \mathbb{M}_{L(\log L), \varphi, \eta}^{i}(\vec{f})(y)>t^{m}\right\}\right)
\end{aligned}
$$

for all $\vec{f}=\left(f_{1}, \cdots, f_{m}\right)$ bounded with compact support.

Proof Similar to the proof of Theorem 3.19 in [8], We can get the desired result by Proposition 2.9, Theorem 3.1 and Corollary 4.1. We omit the details here.

Proposition 4.4 Let $\vec{\omega} \in A_{\overrightarrow{1}}^{\theta}$ and $\eta \geq 2 \theta$. Then there exists a constant $C$ such that

$$
\nu_{\vec{\omega}}\left\{x \in \mathbb{R}^{n}: \mathbb{M}_{L(\log L), \varphi, \eta}^{i}(\vec{f})(x)>t^{m}\right\} \leq C \prod_{j=1}^{m}\left(\int_{\mathbb{R}^{n}} \Phi\left(\frac{\left|f_{j}(x)\right|}{t}\right) \omega_{j}(x) d x\right)^{\frac{1}{m}},
$$

where $\Phi(t)=t\left(1+\log ^{+} t\right)$.

Proof Without loss of generality we may assume $i=1$. Also, by homogeneity, we may also assume that $t=1$. Finally, we may also assume that $\vec{f} \geq 0$. Definite the set 
$\Omega=\left\{x \in \mathbb{R}^{n}: \mathbb{M}_{L(\log L), \varphi, \eta}^{1}(\vec{f})(x)>1\right\}$. It is easy to see that $\Omega$ is open and we may assume that it is not empty (or there is nothing to prove). To estimate the size of $\Omega$, it is enough to estimate the size of every compact set $F$ contained in $\Omega$. We can cover any such $F$ by a finite family of cubes $\left\{Q_{i}\right\}$ for which

$$
1<\frac{1}{\varphi\left(Q_{i}\right)^{\eta m}}\left\|f_{1}\right\|_{L(\log L), Q_{i}} \prod_{j=2}^{m} \frac{1}{\left|Q_{i}\right|} \int_{Q_{i}} f_{j}(x) d x .
$$

Using Vitaly's covering lemma, we can extract a subfamily of disjoint cubes $\left\{Q_{i}\right\}$ such that $F \subset \cup_{i} 3 Q_{i}$. By homogeneity,

$$
1<\frac{1}{\varphi\left(Q_{i}\right)^{\eta m}} \prod_{j=2}^{m}\left(f_{j}\right)_{Q}\left\|f_{1}\right\|_{\Phi, Q_{i}}=\frac{1}{\varphi\left(Q_{i}\right)^{\eta m}}\left\|f_{1} \prod_{j=2}^{m}\left(f_{j}\right)_{Q_{i}}\right\|_{\Phi, Q_{i}} .
$$

We then get

$$
1<\frac{1}{\varphi\left(Q_{i}\right)^{m \eta}\left|Q_{i}\right|} \int_{Q_{i}} \Phi\left(f_{1}(y) \prod_{j=2}^{m}\left(f_{j}\right)_{Q_{i}}\right) d y .
$$

Using now that $\Phi$ is submultiplicative and Jensen's inequality, we get

$$
1<\prod_{j=1}^{m} \frac{1}{\varphi\left(Q_{i}\right)^{\eta}\left|Q_{i}\right|} \int_{Q_{i}} \Phi\left(f_{j}(y)\right) d y .
$$

Thus we get

$$
\varphi\left(Q_{i}\right)^{\eta}\left|Q_{i}\right|<\left(\prod_{j=1}^{m} \int_{Q_{i}} \Phi\left(f_{j}(y)\right) d y\right)^{\frac{1}{m}}
$$

Since $\eta \geq 2 \theta$, we have

$$
\varphi\left(Q_{i}\right)^{2 \theta}\left|Q_{i}\right|<\left(\prod_{j=1}^{m} \int_{Q_{i}} \Phi\left(f_{j}(y)\right) d y\right)^{\frac{1}{m}} .
$$

Since $\vec{\omega} \in A_{\overrightarrow{1}}^{\theta}$, so $\nu_{\vec{\omega}} \in A_{1}^{\theta_{1}}$ by Proposition 2.3. By Proposition 2.2(iii) and the inequality above, we get

$$
\begin{aligned}
\nu_{\vec{\omega}}\left(3 Q_{i}\right) & \leq C \varphi\left(Q_{i}\right)^{\theta} \nu_{\vec{\omega}}\left(Q_{i}\right) \\
& =C \varphi\left(Q_{i}\right)^{2 \theta}\left|Q_{i}\right| \frac{\nu_{\vec{\omega}}\left(Q_{i}\right)}{\varphi\left(Q_{i}\right)^{\theta}\left|Q_{i}\right|} \\
& \leq C\left(\prod_{j=1}^{m} \int_{Q_{i}} \Phi\left(f_{j}(y)\right) d y\right)^{\frac{1}{m}} \prod_{j=1}^{m}\left(\inf _{x \in Q_{i}} \omega_{j}(x)\right)^{\frac{1}{m}} .
\end{aligned}
$$


Finally by the condition on the weights, Hölder's inequality at discrete level and the inequality above, we have

$$
\begin{aligned}
\nu_{\vec{\omega}}(F)^{m} & \leq\left(\sum_{i=1} v_{\vec{\omega}}\left(3 Q_{i}\right)\right)^{m} \\
& \leq C\left(\sum_{i}\left(\prod_{j=1}^{m} \inf _{x \in Q_{i}} \omega_{j}(x) \int_{Q_{i}} \Phi\left(f_{j}(y)\right) d y\right)^{\frac{1}{m}}\right)^{m} \\
& \leq C\left(\sum_{i} \prod_{j=1}^{m}\left(\int_{Q_{i}} \Phi\left(f_{j}(y)\right) \omega_{j}(y) d y\right)^{\frac{1}{m}}\right)^{m} \\
& \leq C \prod_{j=1}^{m} \int_{\mathbb{R}^{n}} \Phi\left(\left|f_{j}(y)\right|\right) \omega_{j}(y) d y,
\end{aligned}
$$

which concludes the proof.

Theorem 4.3 Let $T_{\Sigma \vec{b}}$ be a multilinear commutator with $\vec{b} \in B M O_{\vec{\theta}}^{m}(\vec{\theta}=$ $\left.\left(\theta_{1}, \cdots, \theta_{m}\right)\right)$ and $T$ satisfying (1.1)-(1.4). Let $\vec{\omega} \in A_{\overrightarrow{1}}^{\infty}$. Then there exists a constant $C$ such that

$$
\nu_{\vec{\omega}}\left(\left\{x \in \mathbb{R}^{n}:\left|T_{\Sigma \vec{b}}(\vec{f})(x)\right|>t^{m}\right\}\right) \leq C \prod_{j=1}^{m}\left(\int_{\mathbb{R}^{n}} \Phi\left(\frac{\left|f_{j}(x)\right|}{t}\right) \omega_{j}(x) d x\right)^{\frac{1}{m}},
$$

where $\Phi(t)=t\left(1+\log ^{+} t\right)$.

Proof By linearity it is enough to consider the operator with only one symbol. By homogeneity it is enough to assume $t=1$ and hence we must prove

$$
\nu_{\omega}\left(\left\{x \in \mathbb{R}^{n}:\left|T_{b}(\vec{f})(x)\right|>1\right\}\right)^{m} \leq C \prod_{j=1}^{m}\left(\int_{\mathbb{R}^{n}} \Phi\left(\left|f_{j}(x)\right|\right) \omega_{j}(x) d x\right) .
$$

Now, since $\Phi$ is submultiplicative, we have by Corollary 4.3 and Proposition 4.4,

$$
\begin{aligned}
\nu_{\vec{\omega}} & \left(\left\{x \in \mathbb{R}^{n}:\left|T_{b}(\vec{f})(x)\right|>1\right\}\right)^{m} \\
& \leq C \sup _{t>0} \frac{1}{\Phi\left(\frac{1}{t}\right)^{m}} v_{\vec{\omega}}\left(\left\{x \in \mathbb{R}^{n}:\left|T_{b}(\vec{f})(x)\right|>t^{m}\right\}\right)^{m} \\
& \leq C \sup _{t>0} \frac{1}{\Phi\left(\frac{1}{t}\right)^{m}} \nu_{\vec{\omega}}\left(\left\{x \in \mathbb{R}^{n}: \mathbb{M}_{L(\log L), \varphi, \eta}^{1}(\vec{f})(x)>t^{m}\right\}\right)^{m} \\
& \left.\leq C \sup _{t>0} \frac{1}{\Phi\left(\frac{1}{t}\right)^{m}} \prod_{j=1}^{m} \int_{\mathbb{R}^{n}} \Phi\left(\frac{\left|f_{j}(x)\right|}{t}\right) \omega_{j}(x) d x\right) \\
& \leq C \sup _{t>0} \frac{1}{\Phi\left(\frac{1}{t}\right)^{m}} \prod_{j=1}^{m} \int_{\mathbb{R}^{n}} \Phi\left(\left|f_{j}(x)\right|\right) \Phi\left(\frac{1}{t}\right) \omega_{j}(x) d x \\
\leq C & \prod_{j=1}^{m} \int_{\mathbb{R}^{n}} \Phi\left(\left|f_{j}(x)\right|\right) \omega_{j}(x) d x .
\end{aligned}
$$

The proof is complete. 
Acknowledgments The authors would like to thank the referee for his/her useful comments to improve the paper.

\section{References}

1. Bényi, Á., Torres, R.: Symbolic calculus and the transposes of bilinear pseudodifferential operators. Comm. Par. Diff. Eq 28, 1161-1181 (2003)

2. Bui, T.A.: New class of multiple weights and new weighted inequalities for multilinear operators, to appear in Forum Math (arXiv:1203.2797(2012))

3. Bongioanni, B., Haboure, E., Salinas, O.: Commutators of Riesz transforms related to Schrödinger operators. J. Fourier Anal. Appl 17, 115-134 (2011)

4. Bongioanni, B., Haboure, E., Salinas, O.: Classes of weights related to Schrödinger operators. J. Math. Anal. Appl 373, 563-579 (2011)

5. Coifman, R., Rochberg, R., Weiss, G.: Factorization theorems for Hardy spaces in several variables. Ann. of Math. 103, 611-635 (1976)

6. García-Cuerva, J., Rubio de Francia, J.: Weighted Norm Inequalities and Related Topics. AmsterdamNew York, North-Holland (1985)

7. Grafakos, L., Torres, R.H.: Multilinear Calderón-Zygmund theory. Adv. Math 165(1), 124-164 (2002)

8. Lerner, A.K., Ombrosi, S., Pérez, C., Torres, R.H., Trujillo-González, R.: New maximal functions and multiple weights for the multilinear Calderón-Zygmund theory. Adv. Math 220(4), 1222-1264 (2009)

9. Pérez, C.: Endpoint estimates for commutators of singular integrals. J. Funct Anal 128, 163-185 (1995)

10. Pérez, C., Pradolini, G.: Sharp weighted endpoint estimates for commutators of singular integral operators. Michigan Math. J 49, 23-37 (2001)

11. Pérez, C., Pradolini, G., Torres, R., Trujillo-Gonzalez, R.: End-point estimates for iterated commutators of multilinear singular integrals. Bull. London Math. Soc 46, 26-42 (2014)

12. Pérez, C., Torres, R.: Sharp maximal function estimates for multilinear singular integrals. Contemp. Math. 320, 323-331 (2003)

13. Tang, L.: Weighted norm inequalities for pseudo-differential operators with smooth symbols and their commutators. J. Funct. Anal 262, 1603-1629 (2012)

14. Tang, L., from, E.xtrapolation.: $A_{\infty}^{\rho, \infty}$, Vector-valued inequalities and applications in Schrödinger settings. Ark. Mat 52, 175-202 (2014)

15. Wilson, M.: Weighted Littlewood-Paley Theory and Exponential-Square Integrability, Lecture Notes in Math, vol. 1924. Springer, Berlin (2008)

16. Rao, M.M., Ren, Z.D.: Theory of Orlicz Spaces. Marcel Dekker, New York (1991) 